\title{
Saint Augustin. Un moine devenu Pretre et Evéque
}

\section{In periculo maiore}

$\mathrm{Au}$ mois de septembre 1974 un Colloque a eu lieu à Chantilly sur le thème: "Jean Chrysostome et Augustin". Dans les Actes du Colloque ${ }^{1}$ se trouvent énumérés les centres d'intérêt que voici: le ministère épiscopal des deux hommes et leurs idées à ce propos; la vie monastique; leur recours à la Bible; l' "invention" théologique des deux Pères.

La matinée du 23 septembre fut consacrée à la vie monastique, d'abord d'après saint Augustin, puis d'après saint Jean Chrysostome. J'ai eu alors l'occasion de donner un exposé intitulé: L'utilisation monastique des Actes des Apôtres 4,31,32-35 dans l'oeuvre de saint Augustin ${ }^{2}$. Cette conférence fut suivie d'un exposé de J. - M. Leroux sur Saint Jean Chrysostome et le monachisme, exposé qui trouvait son point culminant dans sa quatrième et dermière partie: Jean Chrysostome et l'institution monastique ${ }^{3}$ : Pour ma part, j'avais surtout insisté sur l'aspect intérieur de la vie monastique augustinienne. J'ai dit notamment que l'anima una de Actes 4,32 est aux yeux d'Augustin l'anima unica Christi ou, ce qui revient au même, l'“âme une de l'Eglise", la koinônia ecclésiale. Dans la conférence de J.- M. Leroux l'aspect intérieur n'était nullement négligé, mais il était plutôt situé au milieu de l'ensemble des considérations sur le monachisme chez Jean Chrysostome.

Après avoir pu relire très attentivement l'exposé de mon partenaire dans les Actes du Colloque, j'ai pensé qu'il serait intéressant

1. Jean Chrysostome et Augustin. Actes du Colloque de Chantilly 22-24 septembre 1974, éd. Ch. KANNENGIESSER (Théologie Historique, 35). Paris, 1975.

2. Jean Chrysostome et Augustin, 93-123.

3. Jean Chrysostome et Augustin, 125-144. 
de compléter mon propre travail en étudiant saint Augustin avec la problématique qui avait guidé les recherches de M. Leroux".

$\mathrm{Au}$ début de sa conférence nous lisons: "...Jean Chrysostome, en quête de perfection, s'adonne successivement aux diverses formes de la vie ascétique: ascèse communautaire dans le cadre de la vie ecclésiale, cénobitisme, anachorétisme; après quelques années de vie mouvementée, il quitte l'institution monastique, apparemment insatisfait des résultats obtenus dans sa quête de l'absolu et il opte, cette fois définitivement, pour l'état ecclésiastique" 5.

Pourquoi cette évolution?

Je résumerais la position de J.- M. Leroux de la façon suivante, en prenant le risque de la durcir quelque peu ${ }^{6}$. J'ai pensé en effet qu'un point de départ ferme me permettrait d'exposer plus clairement l'attitude de saint Augustin en ce même domaine.

4. Plusieurs investigations récentes touchent plus ou moins directement aux mêmes problèmes: H. I. MARROU, Saint Augustin et. la fin de la culture antique (Paris 1938) 360; F. VAN DER MEER, Augustinus de Zielzorger. Utrecht, 1947; M. JOURJON, L'évêque et le peuple de Dieu selon saint Augustin": Saint Augustin parmi nous (1954) 151-178; ID., "Le saint évêque d'Hippone": La tradition sacerdotale. Etudes sur le sacerdoce (1959) 127-141; J. PINTARD, Le sacerdoce selon saint Augustin. Tours, 1960; M. F. BERROUARD, "Saint Augustin et le ministère de la prédication. Le thème des anges qui montent et qui descendent": Recherches Augustiniennes, II (Paris 1962) 447-501; J. GARCIA CENTENO, "El sacerdote como ministro según san Agustín": Revista Agustiniana de Espiritualidad 4 (1963) 375-398; 5 (1964) 234-246; M. PELLEGRINO, Verus sacerdos. Il sacerdozio nell'esperienza e nel pensiero di sant'Agostino. Fossano, 1965: R. CRESPIN, Ministère et sainteté. Pastorale du clergé et solution de la crise donatiste dans la vie et la dottrine de saint Augustin. Paris, 1965; G. MAIOLI di S. TERESA; "Sulla spiritualità sacerdotale ed episcopale in S. Agostino": Scuola Cattolica 93 (1965) 211-222; F. STEGMULLER, Sarcina episcopalis. Zum bischeflichen Ethos des heiligen Augustinus": 'Oberrheinisches Pastoralbaltt 67 (1966) 337-345; A. MANDOUZE, Saint Augustin. L'aventure de la raison et de la grâce (Paris 1968) 121-164; A. ZUMKELLER, Das Mönchtum des heiligen Augustinus 2. Wurzburg, 1968; R. LORENZ, "Zwölf Jahre Augustinusforschung (1959-1970). Mit einigen Nachtragen. XV. Der Seelsorger Augustin": Theologische Rundschau 40 (1975) 229-231; 250-261; A. MANDOUZE, Saint Augustin et le ministère épiscopal; oeuvre citée dans nota 1, pp. 61-73. Mais l'étude le plus directement concernée par notre problème est celle $A$. WUCHERER-HULDENFELD, "Mönchtum und kirchlicher Dienst bei Augustinus nach dem Bilde des Neubekehrten und des Bischofs": Zeitschrift für Katholische Theologie 82 (1960) 182-211. Cette recherche, qui par ailleurs a d'excellentes qualités, se situe trop dans l'antithèse entre la vie contemplative et la vie active, tout en négligeant celle entre le gouvernant et les gouvernés. Il me paraît caractéristique en effet que l'auteur ne fait pas état de ce que Possidius dit sur l'ordination sacerdotale de saint Augustin, ni des Lettres XXI et XXII du dernier.

5. Oeuvre citèe, p. 126. C'est moi qui souligne.

6. Son étude est à lire en effet à la suite de sa première publication dans ce domaine: "Monachisme et.communauté chrétienne d'aprèe saint Jean Chrysostome: Théologie de la vie monastique. Etudes sur la tradition patristique (Théologie, 49) (1961) 143-190. Cet article insiste davantage sur les appréciations positives de J. C. à propos de la vie monastique. 
Jean Chrysostome, semble-t-il, a redouté dans le "monachisme pur", sans charge pastorale, le danger d'une néfaste recherche de soi. La vie monastique risquait de devenir pour lui la quête d'une perfection individuelle stérile, un accomplissement de performances ascétiques comme fin en soi, une existence orgueilleuse et égoïste. La règle fondamentale $d u$ christianisme n'est-elle pas le service d'autrui. Afin d'être totalement au service de ses frères, Jean Chrysostome a donc librement et positivement opté pour le ministère pastoral. Mais cela ne l'a pas empêché de proclamer la nécessité de l'ascèse et de la vie monastique, allant parfois, sur le mode dithyrambique, jusqu'a souhaiter que le monde devienne un vaste monastère. Ce que Jean Chrysostome a voulu combattre, autour de lui et en lui-même, c'était uniquement l'égoïste recherche de soi-même.

En fin de compte, la position d'Augustin a été identique à celle de Chrysostome: c'est l'égoïsme qu'il appréhendait. Mais Augustin a redouté l'egoïsme précisément dans l'exercice autoritaire et prétentieux du sacerdoce et de l'épiscopat, plutôt que dans l'humble existence monastique.

Il reste que saint Augustin a très bien vu les dangers d'une "vie contemplative" pure, c'est-à-dire sans charges pastorales. Il nous a laissé entre autres un Sermon sur la Transfiguration où il apostrophe saint Pierre dans des termes que auraient fait la joie de saint Chrysostome. Et cela parce que Pierre aurait voulu rester près du Seigneur au sommet du mont Thabor.

Saint Pierre a dit à Jésus-Christ: "Maître, il est heureux que nous soyons ici". Il en avait assez de vivre au milieu de la foule, et voilà qu'il venait de découvrir la douce solitude d'une montagne. Le Christ lui-même y servait d'aliment à son esprit. Pourquoi en descendre afin de trouver en bas des labeurs et des douleurs? N'éprouvait-il pas là-haut envers Dieu des amours saints, n'avait-il pas par conséquent des moeurs saintes? C'est son véritable bien qu'il voulait. C'est pour cette raison qu'il a ajouté: "Si tu veux, faisons ici trois tentes, une pour toi, une pour Moïse et une pour Elie" 8 .

Mais saint Augustin n'est pas d'accord avec lui: Descends, Pierre. $\mathrm{Tu}$ voulais te reposer sur la montagne; descends, proclame la parole, insiste à temps, à contretemps, réfute, menace, exhorte, avec une patience inlassable et le souci d'instruire ${ }^{9}$. Travaille, fatigue-toi,

7. Serm. 78: PL 38, 490-493.

8. Serm. 78, 3: PL 38, 491.

9. $2 \operatorname{Tim} 4,2$. 
subis des tourments, afin de parvenir, par la splendeur et la beauté d'une bonne activité, accomplie dans un esprit d'amour chrétien, à posséder ce que figurent les vêtements blancs du Seigneur. Car nous venons d'écouter l'hymme à la charité de l'Apôtre Paul, et c'est là qu'il disait: "La charité ne cherche pas ses propres intérêts" " . Cela veut dire qu'elle partage ce qu'elle possède. Le même Apôtre s'exprime ailleurs d'une façon plus explicite en se décrivant soi-même comme "ne cherchant pas son propre intérêt, mais celui du plus grand nombre, afin qu'ils soient sauvés"

Voilà ce que saint Pierre ne comprenait pas encore quand il désirait rester sur la montagne avec le Christ. Mais, Pierre, le Christ te réservait cela pour après ta mort. Pour le moment il te dit: Descends travailler sur la terre, servir sur la terre, et sur la terre être livré au mépris et à la croix. La Vie elle-même y est descendue pour être tuée; le Pain y est descendu pour avoir faim, la Voie y est descendue pour se fatiguer sur la route; la Fontaine y est descendue pour avoir soif... Et toi, tu refuses le travail? $\mathrm{Ne}$ cherchez pas tes propres intérêts. Aies la charité, proclame la vérité. C'est ainsi que tu parviendras à l'éternité où tu trouveras l'insouciance et la tranquillité ${ }^{12}$.

Les paroles de saint Augustin dans ce Sermon ont sans doute une résonnance bien personnelle. N'était-il pas, lui-aussi, descendu du Thabor pour être au service du troupeau? L'épreuve avait été dure. Dans les Confessions il avoue qu'il avait même pensé à se retirer et se réfugier dans la solitude. Mais il s'était ressaisi en se rappelant une autre parole de saint Paul: "Le Christ est mort pour tous, afin que les vivants ne vivent plus pour eux-mêmes, mais pour celui qui est mort et ressuscité pour eux ${ }^{13}$.

Dans le très long mais aussi très intéressant livre XXII de son Contra Faustum (le septième oeuvre que saint Augustin a mise en chantier après sa consécration épiscopale), il répond aux critiques que l'évêque manichéen Faustus avait formulées à l'égard de plusieurs personnages de l'Ancien Testament, de leurs "actions scandaleuses et leur vies infâmes", flagitia et turpes vitae ${ }^{14}$. Il va sans dire que Faustus n'a pas manqué par exemple de parler avec ironie des

10. 1 Cor 13, 5 .

11. 1 Cor 10, 33.

12. Serm. 78, 6: PL 38, 492-493.

13. Confessiones X, 43: PL 32, 808; éd. M. SKUTELLA, p. 262-263; 2 Cor $5,15$.

14. Contra Faustum XXII, 1: PL 42, 401; CSEL 25, 591. 
aventures conjugales de Jacob. Sans s'en rendre compte, celui-ci avait épousé Léa au lieu de sa soeur Rachel, laquelle est devenue quand même sa femme sept ans plus tard. Les deux soeurs, et leurs servantes respectives Zilpa et Bilha, ont donné à Jacob, à part sa fille Dina, les douze fils qui allaient devenir les pères des douze tribus d'Israël ${ }^{15}$. Augustin explique que tous les faits et gestes des patriarches avaient un sens prophétique et annonçaient symboliquement les réalités de la Nouvelle Alliance. Son explication des aventures matrimoniales de Jacob est aussi compliquée que ces péripéties elles-mêmes. Ramenons-la à l'essentiel. Ces événements nous parleraient symboliquement de la vie contemplative, indiquée par Rachel, qui était radieuse de beauté, et de la vie active, indiquée par Léa dont les yeux étaient ternes.

Les notions de "vie contemplative" et de "vie active" sont complexes chez saint Augustin ${ }^{16}$, comme ailleurs. Mais dans les passages qui nous intéressent directement dans le présent exposé, la "vie contemplative" est une vie sans aucune charge pastorale, une vie purement monastique, faite de prières, de réflexión, d'étude. La "vie active" au contraire y est le ministère d'un évêque ou d'un autre "clerc".

Ces derniers, dit Augustin, sont des gens pleins d'activité. En vue du bien du grand nombre ils se donnet beaucoup de mal dans la conduite du troupeau. Ils ont à jouer un rôle influent et leur prestige est cher aux fidèles. Mais s'ils sont ce qu'ils doivent être, ils ne manqueront pas de rendre témoignage à cette autre facon de vivre qui, libre de toute charge pastorale, cherche à atteindre et à "regarder" la Vérité. Il y a beaucoup de gens de talent et d'une ardeur chaleureuse qui évitent l'activité sacerdotale à cause des agitations qu'elle comporte; de tout leur coeur ils cherchent à être libres pour apprendre; ils se jettent pour ainsi dire dans les bras de la rayonnante Rachel, comme Jacob. Que les pasteurs en activité rendent à cette oisiveté bien remplie un bon témoignage. Les fidèles en prendront ainsi connaissance et elle obtiendra ainsi chez eux la bonne réputation qu'elle mérite ${ }^{17}$. Mais -et ici nous arrivons à ce qui nous importe le plus pour le moment- cette bonne réputation perd sa justification quand la "vie contemplative" retient égoïstement dans l'oisiveté un homme que pourrait fort bien diriger une

15. Gen 29, 30

16. Volr A. MANRIQUE, La vida monástica en san Agustín (El EscorialSalamanca 1959) 362-363, note 561 .

17. Contra Faustum XXII, 56: PL 42, 436; CSEL 25, 652. 
Eglise et l'empêche de prendre part à l'administration qui se fait en vue du bien commun. Il y a des moines capables, sachant agir, dignes d'être chargés du gouvernement d'une Eglise pour y administrer les mystères de la foi; ils ont de l'ardeur pour la connaissance de la vérité, pour la recherche et la contemplation de la sagesse; mais il ne faudrait pas qu'à cause de cela ils désirent se tenir à l'écart des désagréments de la vie pastorale et se cacher dans une oisiveté où l'on vit uniquement pour apprendre et pour instruire... ${ }^{18}$. Il faut que la "vie contemplative" garde une bonne réputation. Ceux qui peut-être avaient décidé de rester libres afin de se consacrer à l'étude, doivent pour cette raison bien accepter de faire l'expérience douloureuse du pastorat et d'en assumer la charge pour le bien de toute la communauté chrétienne. A condition, bien entendu, qu'ils sachent se donner de la peine et soient ainsi aptes à gouverner les fidèles. De cette façon, la connaissance de la sagesse, pour laquelle ils avaient voulu vivre en toute liberté, ne sera pas traitée de manière blasphématoire par des fidèles moins bien informés. Elle obtiendra de leur part cette bonne renommée qui est indispensable pour encourager les adhérents de la vie monastique pure qui seraient encore en formation ${ }^{19}$.

Est-ce à dire que cette "vie contemplative" devrait pratiquement rester réservée à ceux qui sont incapables de mener un autre genre de vie, et que tous les hommes doués devraient se précipiter pour se lancer dans la vie pastorale? Non pas! Saint Augustin va dire maintenant brièvement ce qu'il répétera avec plus d'insistance ailleurs: Pour le dire bien clairement, ils doivent assumer les charges de la vie pastorale uniquement s'ils y sont contraints par la force des circonstances.... ${ }^{20}$.

Dans le monde entier on peut voir des gens quitter leurs activités dans le "siècle" et entrer dans cette existence libre où l'on vit pour apprendre la vérité, pour la "regarder", pour embrasser Rachel. Mais voilà qu'inopinément ils sont captés par un besoin d'Eglise et rangés avec les "travailleurs". Léa n'a-t-elle pas dit à l'époux de Rachel: "Il faut que tu viennes vers moi"? Et Jacob ne lui a pas refusé sa nuit. En effet, dans la nuit du temps présent les pasteurs engendrent des enfants: les fidèles. C'est pourquoi les fidèles reconnaisants disent du bien de la vie monastique pure. Ils se rendent compte que c'était par amour pour ce genre de vie que tel ou tel de

18. Contra Faustum XXII, 56: PL 42, 436; CSEL 25, 652-653.

19. Contra Faustum XXII, 58: PL 42, 436-437; CSEL 25, 653.

20. Contra Faustum XXII, 58: PL 42, 437; CSEL 25, 654. 
leurs évêques avait jadis changé son existence en renonçant à tout espoir dans le "siècle" et en devenant moine. Mais on était venu le tirer de là pour qu'il accomplisse une oeuvre de miséricorde, la direction des fidèles. Au beau milieu de leurs labeurs, ces pasteurs visent maintenant à rendre plus largement et plus lucidement apprécié et connu cet état de vie auquel ils s'étaient jadis convertis ${ }^{21}$.

Procédons à une première récapitulation. 1. Saint Augustin, donc, a très bien vu, comme saint Jean Chrysostome, quel danger d'égoïsme peut se cacher dans une vie monastique pure, c'est-à-dire sans charge pastorale. 2. Il estimait que les moines-laïques ne devaient pourtant pas assumer les charges d'un pastorat sans y être invités par l'Eglise. 3. Une fois mis à la tête d'une Eglise locale, l'ancien moine-laïque devait faire tout son possible pour susciter chez ses fidèles du respect et de l'amour pour la vie purement contemplative qu'il avait quittée pour se mettre à la disposition de l'Eglise et au service des fidèles.

Saint Augustin aimait donc beaucoup la vie monastique pure, tandis qu'il appréhendait certains dangers précisément du côté du sacerdoce. C'est ce qui deviendra plus compréhensible par la suite.

C'est vers la fin du IVe siècle également, semble-t-il ${ }^{22}$, que saint Augustin a été consulté par le prêtre Eudoxius et les moines qui vivaient avec lui sur l'île de Capraria. Ils désiraient savoir comment se comporter quand un moine-laîque était sollicité pour prendre en charge une Eglise locale. La réponse d'Augustin fut très nuancée. C'est pourquoi nous allons rencontrer cette réponse -la Lettre XLVIII de saint Augustin- à plusieurs endroits successifs du présent exposé. Pour l'instant je veux souligner seulement que saint Augustin, qui a tant estimé et aimé la quête de Dieu dans une vie de prière et d'étude, a fait remarquer avec insistance qu'on peut mener cette vie bien égoïstement. Il affirme en effet: les moines de Capraria ne devaient pas refuser l'épiscopat, le sacerdoce ou le diaconat en vertu des charmes d'une vie "assise", blandiente desidia, ces charmes qui pourraient bien les engloutir dans la mollesse engourdie d'une existence sans ocupation, torpida uacationis mollitia. Il ne faut pas préferer ses aises personnelles aux besoins de l'Eglise. Si aucun homme de bien ne voulait assister l'Eglise dans ses peines d'accouchement, elle serait dans l'impossibilité de mettre au monde des enfants. Dans la vie monastique c'est comme partout:

21. Contra Faustum XXII, 58: PL 42, 437; CSEL 25, 654.

22. Voir CSEL 58, 18. 
qu'on se tienne à distance d'agréments purement et simplement humains. Qu'on se rende compte qu'il n'y a aucun endroit où le Mauvais ne puise tendre ses pièges ${ }^{23}$.

Une vingtaine d'années plus tard, saint Augustin a rédigé le livre dix-neuvième de la Cité de Dieu. Dans le dix-neuvième chapitre de ce livre il traite des trois genres de vie, le genus otiosum, le genus actuosum et le genus ex utroque compositum (c'est-à-dire l'otium combiné avec une dignitas ou fonction publique, le fameux otium cum dignitate.) La terminologie est empruntée de l'éthique politique de l'Antiquité ${ }^{24}$, mais la fonction, la dignitas, dont il est question ici est le ministère pastoral d'un évêque ou d'un autre "clerc".

Dans le genus otiosum, sans ministère pastoral, il ne faut pas oublier de penser à son prochain. Dans ce genre d'existence ce n'est pas l'iners uacatio, l'inactivité et la liberté de toute charge publique, qui doit faire plaisir, mais la recherche et la découverte de la vérité, le progrès personnel dans ce domaine et le partage qu'on fait avec autrui de la vérité découverte. C'est l" "amour de la vérité qui doit animer l'otium sanctum ${ }^{25}$, le loisir saint ${ }^{26}$. L'"amour de la véritè"... Il est intéressant que saint Augustin ne dit pas amor ueritatis, mais caritas ueritatis ${ }^{27}$. Il ne s'agit pas de l'étude aride de vérités abstraites, mais de la connaissance progressive d'une t̂tre aimé, partagée avec d'autres à cause du même amour. Autrement dit, la vie monastique pure n'est pas à regarder comme une existence dépourvue d'activité. Sans être chargée d'un ministère public, clérical, elle a bien son activité à elle. Nous y reviendrons longuement plus loin.

Saint Augustin, encore moine-laïque, est devenu lui-même prêtre d'abord, evêque ensuite. Mais on dirait qu'il savait alors retrouver pendant la nuit le genre de vie qu'il ne pouvait plus exercer pendant ses journées. C'est tout seul, à la lumiére de sa lampe, qu'il faisait ses réflexions théologiques ou corrigeait ce qui était déjà découvert, dicté et mis au net. Il retrouvait ainsi son otium, mais,

23. Ep. 48, 2: PL 33. 188; CSEL 34, 2, 138.

24. Voir J.- M. ANDRE, L'Otium dans la vie morale et intellectuelle romaine des origines à l'époque augustéenne. Paris, 1966.

25. Sans etre devenu un terme technique dans ce sens, sanctus a chez Augustin souvent une connotation monastique. Cela semble bien être le cas ici également.

26. PL, 647; CC 48, 686-687.

27. Saint Augustin approfondit ainsi sa pensée. Au début du passage il parlait encore de amor ueritatis. 
pour reprendre les termes de la Cité de Dieú, cette oisiveté n'était pas une iners uacatio et même pendant la nuit Augustin pensaitau partage avec autrui de la vérité ${ }^{28}$.

Il était d'ailleurs très conscient d'avoir gardé son coeur de moine-laïque au beau milieu de ses occupations pastorales. Dans un Sermon prononcée à l'occasion d'un anniversaire de sa propre consécration épiscopale il a dit: J'ai pris sur moi la charge de l'épiscopat. Mais personne ne l'emporterait sur moi en ce qui concerne la vie totalement libre, sans aucun souci pastoral: rien n'est meilleur, rien n'est plus doux que d'explorer le trésor divin, sans agitation autour de nous. C'est doux, c'est bon... ${ }^{29}$.

Une fois devenu évêque, l'ancien moine-laïque doit faire tout son possible pour faire estimer et aimer autour de lui la vie monastique pure qu'il a dû quiter. Saint Augustin, nous l'avons vu, ecrivait cela dans son Contra Faustum. Mais comme si souvent, en disant cela saint Augustin parlait en premier lieu à lui-même. Pour que le lecteur se rende compte de la façon dont Augustin lui-même a su communiquer à ses ouailles l'amour qu'il avait gardé pour la vie monastique pure, il faudrait citer intégralement le très beau Traité 57 sur l'Evangile selon saint Jean. J'en donnerai ici quelques extraits.

Les Traités 56, 58 et 59 parlent de Jean 13, 1-20, c'est-à-dire du lavement des pieds et de la réaction bien connue de saint Pierre à ce propos. Dans le Traité 57, saint Augustin s'arrête un moment pour faire des réflexions très personnelles. Le lavement des pieds l'a fait penser à une phrase du Cantique des Cantiques - il s'agit de 5,3: "J'ai lavé mes pieds, comment pourrais-je les salir de nouveau?" Ce sont là des paroles de l'Epouse. Saint Augustin les met dans la bouche de l'Eglise.

"J'ai lavé mes pieds, comment pourrais-je les salir de nuoveau?" L'Eglise parle ainsi entre autres dans ceux de ses membres qui sont suffisamment purs pour pouvoir dire: "J'ai le désir de m'en aller et d'être avec le Christ, mais il est plus urgent pour votre bien que je demeure dans la chair" ${ }^{30}$. L'Eglise le dit aussi dans ceux de ses membres qui prêchent le Christ et lui ouvrent l'entrée dans les coeurs des hommes pour qu'il y habite par la foi ${ }^{31}$. L'Eglise parle ainsi en-

\footnotetext{
$128-130$.

28. POSSIDIUS, Vita s. Aug. 24, 11: PL 32, 54; éd. M. PELLEGRINO, p.

29. Sermo Frangipane II (339), 4: PL 46, 960; $\mathrm{MA} \mathrm{I,} 193$.

30. Fil. 1, 23-24.

31. Ep. 3, 17.
} 
fin dans des gens qui se demandent s'ils doivent supporter d'être chargés d'un ministère, s'estimant peu capables de l'exercer sans culpabilité et craignant que, tout en prêchant aux autres, ils ne soient eux-mêmes disqualifiés ${ }^{32}$. En effet, on est bien plus en sûreté en écoutant qu'en prêchant la vérité. Car lorsqu'on est simple auditeur, on garde l'humilité, mais quand on prêche la vérité, il est presque impossible à un homme, quel qu'il soit, de se garder de vanité, au moins d'un tout petit peu de vanité, mais d'une vanité suffisante pour se salir les pieds. C'est pour cette raison que l'Eglise dit par leur bouche: "J'ai lavé mes pieds, comment pourrais-je les salir de nouveau?" ${ }^{33}$.

Plus loin saint Augustin reprend son idée d'après laquelle on garde l'humilité quand on reste simple auditeur. Il la développe maintenant en disant: Jouissons donc du plaisir d'écouter lorsque la vérité nous parle intérieurement sans aucun bruit. Quand la vérité se fait entendre de l'extérieur par l'intermédiaire d'un clerc qui lit tel passage biblique, ou d'un autre qui en fait l'introduction, ou qui prononce un sermon là-dessus, ou qui donne un exposé, ou qui formule des préceptes, des paroles de consolation et d'encouragement, voire par l'intermédiaire du jeune chantre qui fait entendre un psaume, alors, il faut bien l'avouer, ceux qui s'acquittent de ces offices ont à craindre de salir leurs pieds: l'amour des éloges humains risque de se glisser dans leur coeur et de leur inspirer le désir de plaire aux hommes. Mais celui qui les écoute dans un sentiment de joie et de "piété" 3 'a aucune raison pour tirer vanité de ce que d'autres qu'eux accomplissent, et bien loin de s'enfler d'orgueil, c'est d'un coeur humble qu'il se réjouit d'entendre la voix de la vérité du Seigneur ${ }^{35}$.

Après ces paroles générales, saint Augustin se met à faire sentir son amour pour la vie monastique pure, vie où l'on écoute beaucoup, mais parle peu.

La sainte ${ }^{36}$ Eglise se tient tranquillement et savoureusement recueillie dans ceux de ses membres qui savent écouter avec joie et humilité, et qui mènent une vie sans agitation avec des occupations douces, centrées sur le salut éternel. Là, que la sainte Eglise déclare,

32. Cf. 1 Cor. 9, 27.

33. In Iohannis evangelium tr. 57, 2: PL 35, 1790; CC 36, 470.

34. A propos de la pietas voir mon article "Ecouter la parole de Dieu sant interrompre ni protester": Augustiniana, 27 (1977) p. 125-149.

35. In Iohannis evangelium tr. 57, 3: PL 35, 1791; CC 36, 470-471.

36. Sancta ecclesia... Voir note 25 . 
avec l'Epouse du Cantique: "Je dors, mais mon coeur veille" "Q Que veut-elle dire par cela? On va vous le dire: Je me repose en écoutant. Ma tranquillité n'entend nullement alimenter je ne sais quelle inaction; elle veut recueillir la sagesse. "Je dors; mais mon coeur veille", je suis libre de toute autre occupation et ce que je vois c'est que tu es le Seigneur. Car la sagesse du scribe s'acquiert aux heures libres, et celui qui diminue ses agitations la recueillera ${ }^{38}$. "Je dors, mais mon coeur veille", je me tiens en repos, loin des tracas absorbants, et mon ame tourne toute son attention vers ce Dieu qui nous aime ${ }^{39}$.

Mais l'évêque Augustin qui exprime ainsi son amour pour la vie monastique absolue, et que ses fidèles ont sans doute écouté avec joie et sympathie, avait un jour quitté ce "Thabor". L'Epoux de l'Eglise était venu frapper à sa porte en disant: "Ce que je vous dis dans les ténèbres, dites-le au grand jour, et ce que vous entendez dans le creux de l'oreille; proclamez-le sur les toits" ${ }^{40}$. La voix de l'Epoux se faisait entendre à la porte; elle parlait avec force: "Ouvre-moi, ma soeur, ma proche, ma colombe, ma parfaite. Car ma tête est couverte de rosée froide, mes boucles ruissellent des gouttes glaciales de la nuit" ${ }^{41}$. C'est comme si l'Epoux disait à l'Eglise: Tu te gardes libre de toute autre occupation, et ainsi tu me tiens la porte fermée; tu t'appliques à procurer de la tranquillité à quelques-uns de tes membres, tandis que l'iniquité, qui abonde, refroidit la charité du grand nombre. En effet, dans ces paroles de l'Epoux, la nuit est l'iniquité; la rosée et les gouttes glaciales indiquent ceux qui deviennent froids et qui tombent; ils refroidissent la tête de l'Eglise; c'est-à-dire: ils sont cause que Dieu n'est plus aimé. Car la tête de l'Epoux c'est Dieu ${ }^{42}$. Le divin Epoux frappe donc à la porte de l'Eglise pour tirer de leur tranquillité les "saints oisifs" ${ }^{43}$ et crie: "Ouvre-moi, toi qui es ma soeur en vertu de mon sang; qui est ma proche parce que je me suis approché de toi; qui es ma colombe, esprit de l'Esprit; qui es ma parfaite, grâce à tout ce que je t'ai dit lors de ton repos, quand tu m'écoutais d'une façon plus entière que les autres. Ouvremoi, prêche-moi. Car comment pourrais-je entrer chez ceux qui m'ont fermé leur porte, si personne n'est là pour m'ouvrir? Comment pourraient-ils m'entendre sans prédicateur? ${ }^{45}$.

\footnotetext{
37. Cant. 5, 2.

38. Cf. Qoh 38, 25 .

39. PL 35, 1791; CC 36, 471.

40. Mt. 10, 27.

41. Cant. 5, 2.

42. Cf. 1 Cor 11, 3 .
} 
Ceux qui aiment cette vie libre où l'on vaque à de si bonnes occupations, qui ne désirent pas afronter les embarras laborieux du ministère actif, voửraient bien que les Apôtres et les prédicateurs de jadis reviennet pour lutter contre le Mal et contre le refroidissement de l'amour. Mais ils doivent comprendre que les Apôtres et leurs compagnons ont disparu définitivement. Bien sûr, on, les comprend bien, ces gens-là: ils se sentent peu aptes à assumer les charges du ministère et à les accomplir sans blâme. L'Eglise les regard. Ils sont tant bien que mal capables de prêcher, de gagner des fidèles et de les gouverner; mais ils craignent de pècher dans lexercice d'une activité aussi difficile. Alors l'Eglise dit en leur nom: "J'ai lavé mes pieds, comment pourrais-je les salir de nouveau?" Car celui qui ne commet pas d'écart dans ses paroles, c'est un homme parfait ${ }^{46}$. Et qui est parfait, qui est celui qui ne pèche pas au milieu de tant d'iniquité et d'un si grand refroidissement de l'amour? "J'ai lavé mes pieds, comment pourrais-je les salir de nouveau?" Je lis, j'entends, en effet: "Ne soyez pas nombreux, mes frères, à devenir docteurs, parce que vous n'en recevrez qu'un jugement plus sévère; car à beaucoup d'égards nous commettons des écarts, tous sans exception" ${ }^{47}$. Et l'Eglise répète encore une fois en leur nom: "J'ai lavé mes pieds, comment pourrais-je les salir de nouveau?" ".

Mais alors elle se ressaisit, la crise passe, et elle dit: Cependant voici que je me lève et que je vais ouvrir la porte au christ. Mes pieds, salis de nouveau, ô Christ, lave-les: pardonne-nous nos péchés car notre amour n'est pas éteint et nous-aussi, nous pardonnons à ceux qui nous ont offensés. Lorsque nous t'écoutons, ô Christ, alors tout humblemente nous tressaillons d'émotion avec toi dans les cieux. Mais lorsque, pour t'ouvrir la porte, nous te prêchons, nous foulons la terre avec nos pieds. Si l'on nous critique, nous sommes troublés; si l'on nous aprouve et fait notre éloge, cela nous enfle d'orgueil. Lave donc nos pieds qui étaient déjà propres mais que nous avons salis de noveau en marchant sur la terre, et cela pour aller t'ouvrir la porte ${ }^{4}$.

Si nous avons peut-être commis des écarts en parlant, mes frè-

43. Sanctis otiosis... Voir note 25 .

44. In Iohannis evangelium tr. 57, 4: PL 35, c. 1791; CC 36, p. 471.

45. Cf. Rom. 10, 14.

46. Jue 3,2 .

47. Jue 3, 1-2 472.

48. In Iohannis evangelium tr. 57, 5-6: PL 35, c. 1791-1792; CC 36, p. 471-

49. In Iohannis evangelium tr. 57, 6: PL 35, c. $1792 ;$ CC 36, p. 47. 
res, ou si nous avons été trop orgueilleusement sensibles à vos applaudissement, obtenez-nous de Dieu, par des prières qui lui plaisent, qu'il digne purifier nos pieds ${ }^{50}$.

Rappelons-nous la raison pour laquelle ce texte magnifique a été cité. Dans son Contra Faustum saint Augustin a affirmé qu'un évêque qui a quitté la vie monastique pure afin de se charger de la direction d'une Eglise locale, doit faire tout son possible pour faire estimer et aimer cette vie monastique "contemplative". Le Traité 57 sur l'Evangile selon saint Jean nous a permis de voir comment saint Augustin lui-même s'est acquitté de ce devoir auprès des fidèles.

Mais le texte que nous avons lu dépasse largement le service qui lui était demandé. Aussi allons-nous de nouveau nous interrompre pour faire le point:

1. Saint Augustin a très bien vu les éventueles dangers de la vie monastique absolue, sans charge pastorale: on peut la vivre égoïstement. 2. Il estimait néanmoins que les moines-laïques ne devaient pas assumer un ministère pastoral sans y être invités par l'Eglise. 3. Une fois mis à la tête d'une Eglise locale, l'ancien moine-laïque devait faire tout ce qu'il pouvait pour susciter chez les fidèles du respect et de lamour pour la vie purement "contemplative", vie qu'il avait quittée pour se mettre à la disposition de l'Eglise-génitrice-de-fidèles et au service de ses ouailles. C'est dans ce sens que l'ancien moine-laïque Augustin a parlé dans le Traité 57 sur l'Evangile selon saint Jean. Avant de lire ce Traité, nous avons déjà énuméré ces trois points lors d'une première récapitulation. Maintenant nous pouvons ajouter quelques précisions complémentaires:

4. La vie monastique purement "contemplative" n'est pas une iners uacatio. "Je dors, mais mon coeur velle", disait l'Epouse du Cantique. L'amour de la vérité, la caritas ueritatis, pousse à une "activité" intense, la quête de la sagesse. Saint Augustin, évêque plein de zèle pendant ses journees, "redevenait moine-laïque" pendant ses nuits. 5. C'est encore dans un autre sens que la vie monastique pure n'est pas une iners uacatio. La Vérité qu'on découvre n'est pas à garder pour soi, comme une possession privée, mais à partager avec autrui. Malgré l'absence de fonctions pastorales, certains moines-laïques doués ont des devoirs d'ordre doctrinal envers leurs prochains, ceux de leur maison et ceux du dehors. 6. Les moines-laïques que l'Eglise appelle au ministère pastoral ont à redou-

50. In Iohannis evangelium tr. 57, 6: PL 35, 1792; CC 36, 472. 
ter, d'après saint Augustin, un double danger: d'abord celui de vouloir plaire aux fidèles, orgueilleusement; ensuite celui de la crainte de déplaire aux pécheurs parmi eux.

Les trois derniers points de cette récapitulation seront mis en pleine lumière par les textes qu'il nous reste de parcourir.

Aux yeux de saint Augustin, la vie monastique pure n'es donc pas $\mathrm{du}$ tout une petite vie tranquille et confortable. On risque de le penser à cause d'un soi-disant "platonisme" qu'on lui attribue avec un peu trop de facilité.

Nous avons déjà rencontré la Lettre XLVIII de saint Augustin, adressée à Eudoxius et les moines-laïques qui habitaient avec lui sur l'île de Capraria. Une bonne partie de cette Lettre est consacrée à l'évocation de leur vie quotidienne, une existence "contemplative", sans aucune activité pastorale. Or, chose curieuse et très intéressante, toute la description qu'Augustin donne de ce genre de vie, est structurée autour de l'idée d'activité, de travail.

Eudoxius et ses frères, dit Augustin, doivent accomplir leur travail à eux intégralement pour la gloire de Dieu qui, lui, opère tout en tous; qu'ils soient fervents d'esprit, pour que leur âme soit louée dans le Seigneur. C'est dans ces dispositions qu'ils doivent agir activement et avec ardeur, travailler d'une façon infatigable dans leur chantier à eux: celui des prières, des jeûnes, de la bienfaisance. Dans ce dernier domaine, il y a deux sortes d" "aumônes". Il peut s'agir bien entendu des secours matériels à procurer aux indigents du dehors, mais aussi bien du pardon mutuel entre les frères. Leur chantier à eux est aussi celui où l'on essaie de triumpher de ses néfastes habitudes, où l'on "meurtrit son corps et le traîne en esclavage" ${ }^{51}$, où l'on supporte patiemment les tribulations, et surtout où l'on se supporte mutuellement avec cet amour qui fait qu'on se dépasse - car que supporterait celui qui ne supporte pas son frère?-. Les frères de Capraria ont encore un autre labeur à accomplir: ils ont à discerner les pièges que le Tentateur a tendus avec adresse et, en prenant le bouclier de la foi, à repousser et éteindre ses traits enflammés. Et puis ils ont le travail qui plus tard sera appelé précisément l'opus dei, celui de chanter, et de célébrer le Seigneur, soit silencieusement dans leur coeur, soit avec des voix qui ne sont pas en disharmonie avec l'intérieur ${ }^{52}$. Toute cette activité, qu'ils s'en

51. 1 Cor $9,27$.

52. Dans la Règle de saint Augustin se lit une pharase comparable. Voir Praeceptum, lignes 41-42: "Psalmis et hymnis cum oratis deum, hoc uersetur in corde quod profertur in uoce". 
acquittent pour la gloire de Dieu. C'est-là le travail propre au chemin qui conduit tout droit vers la vie éternelle. Pendant la marche on lève constamment les yeux vers le Seigneur, car c'est lui qui tire nos pieds du filet ${ }^{53}$. Cette activité du monachisme pur est une activité qui n'est ni surchauffée par toute sorte d'affaires, ni refroidie par l'inactivité; elle n'est ni turbulente, ni engourdie, ni téméraire, ni fuyarde; ni précipitée, ni endormie. Que telle soit l'activité "immanente" d'Eudoxius et des siens dans leur retraite insulaire: alors le Dieu de la paix ${ }^{54}$ sera avec eux ${ }^{55}$.

Non pas une iners uacatio donc. Ailleurs on retrouve des perspectives identiques, mais présentées d'une façon plus serrée et par conséquent moins évocatrice.

Possidius, le biographe de saint Augustin, décrit ainsi les trois années qu'Augustin et quelques compagnons ont passées dans sa maison paternelle à Thagaste, après son retour d'Italie: il était détaché des soucis de ce siècle et vivait pour Dieu dans des jeûnes, des prières et de bonnes oeuvres, réfléchissant jour et nuit sur "la loi de Dieu" ${ }^{56}$. Le texte de Possidius est un peu plus long en réalité; nous lirons la suite dans quelques instants.

On s'est posé la question de savoir si la maison de Thagaste était déjà un monastère au sens strict du terme et on y a répondu diversement ${ }^{57}$. Pour ma part j'estime évident que de toute façon la vie d'Augustin et de ses amis à Thagaste ressemblait déjà beaucoup à celle des moines de Capraria, évoquée dans la Lettre XLVIII.

Il y a une description du même genre dans le Commentaire du Psaume 99,12 et le terme de "monastère" s'y trouve en toutes lettres. Saint Augustin y parle de cette "vie communautaire que des "frères" mènent dans un monastère", in monasterio. Ce sont là, dit-il, des hommes admirables, des hommes saints ${ }^{58}$, qui pasent chacun de leurs jours dans le chant d" "hymnes", en priant, en louant Dieu; c'est de cela qu'ils vivent; ils vaquent à la lecture; ils travaillent de leurs mains et pourvoient ainsi à leur subsistence; vivant sans avidité ils ne demandent rien; tout ce qu'ils reçoivent de la part de Chrétiens dévoués, ils en usent avec charité et en proportion de

53. Ps. 24, 15.

54. 2 Cor 13,11

55. Ep. 48, 3: PL 33, 188-189; CSEL 34, 2. p. 48.

56. POSSIDIUS, Vita s. Aug. 3, 1-2: PL 32, c. 36; éd. M. PELLEGRINO,

57. Voir A. MANDOUZE, oeuvre citée dans note 4, p. 200-209 et A. ZUMKELLER, oeuvre citée dans la même note, p. 42-44.

58. Sancti... Voir note 25. 
leurs besoins; nul ne s'arroge une chose qu'un autre n'ait pas; ils s'aiment tous, tous se supportent mutuellement ${ }^{59}$. Mais saint Augustin ne manque pas d'ajouter que la réalité n'est pas toujours aussi belle ${ }^{60}$.

Dans son opuscule sur Le travail des moines, saint Augustin affirme qu'il voudrait bien personnellement, comme les moines sans charge pastorale, faire chaque jour un peu de travail manuel à des heures déterminées et pouvoir disposer du reste de son temps pour lire et prier, ou pour "accomolir quelque chose à propos de l'Ecriture sainte", aliquid de diuinis litteris agere ${ }^{61}$. Ces derniers termes ne sont pas bien clairs. Mais rappelons-nous les deux raisons pour lesquelles la vie monastique pure n'est pas, d'après saint Augustin, une iners uacatio: d'abord, le travail de sanctification est un véritable et dur travail; puis, celui qui a pu pénétrer dans la vérité ne doit pas la garder comme une possession privée mais accomplir un travail de partage. C'est peut-être à cela que font allusion les paroles aliquid de diuinis litteris agere.

Reprenons maintenant la lecture de la description que Possidius a donnée de la vie d'Augustin et ses amis à Thagaste, lecture que nous avons provisoirement interrompue un peu plus haut. Détaché des soucis de ce siècle, Augustin vivait pour Diev dans des jeûnes, des prières et de bonnes oeuvres, réfléchissant jour et nuit sur "la loi de Dieu". C'était sant doute l'Ecriture sainte. Puis Possidius poursuit: Pendant ses réflexions et ses prières, Dieu lui fit comprendre certaines choses et Augustin les faisait connaitre aux présents et aux absents par des entretiens et des écrits ${ }^{62}$. Encore une fois, serait-ce cela, cet aliquid de diuinis litieris agere?

Quoi qu'il en soit, la même idée d'une vie monastique pure, sans activité pastorale mais avec une certaine activité dans le domaine de la communication de la vérité se manifeste dans la Lettre XCV de saint Augustin, adressée à Paulin de Nole. Augustin y parle de l'otium christianum, une vie libre de toute charge pastorale et centrée sur le Christ, et libre pour recueillir la sagesse chrétienne et éventuellement pour l'exposer, ad christianam sapientiam percipiendam vel dis sere nda m. ${ }^{63}$.

Somme toute, aux yeux de saint Augustin la vie monastique absolue n'est pas une existence de tout repos. Le dur labeur de la

59. Enarrationes in Ps: 99, 12: PL 37, 1278; CC 39, 1401.

60. Enarrationes in Ps. 99, 12: PL 37, 1278-1279; CC 39, 1401.

61. De Opere monachorum 29. (37): PL 40, 576; CSEL 41; 586-587.

62. POSSIDIUS, Vita s. Aug. 3, 2 : PL 32,36 ; éd. M. PELLEGRINO, p. 48.

63. Ep. 95, 9: PL 33, 356; CSEL 34, 2, 513. 
sanctification de tous et le partage avec autrui des valeurs chrétiennes qu'on a découvertes y remplissent les journées, voire les nuits. A condition bien entendu que cette existence se déroule comme il faut. Les dangers de paresse, de fainéantisme, de somnolence spirituelle qui s'y attachent n'ont en effet pas échappé à saint Augustin.

Mais à ses yeux la uita actuosa, c'est-à-dire la vie pastorale d'un prêtre, par exemple, ou d'un évêque, constituait un danger bien plus grand. C'est là qu'on se trouve in periculo maiore, pour reprende les temes du titre de cette recherche.

Afin de nous rendre compte de ce que le ministère pastoral représentait pour saint Augustin, nous allons lire tout d'abord sa Lettre XXI, Lettre qu'il a adressée à Valerius son évêque, peu de temps après son accession forcée au sacerdoce.

Saint Augustin n'a en effet nullement désiré cette "fonction". Elle lui a été imposée. Il est vrai qu'il l'a acceptée et qu'il a su quitter Dieu pour Dieu, mais il l'a fait en pleurant de chaudes larmes. On connaît les faits. Il se trouvait de passage à Hippone, en 391. A cette période de sa vie, il évitait de se rendre dans une ville dont le siège épiscopal était vacant. Mais à Hippone il pensait n'avoir à craindre: Hippone avait son évêque. Il s'appelait Valerius. Ce nom ne nous dirait sans doute rien, si Valerius n'àvait pas été le prédécesseur immédiat d'Augustin au siège épiscopal d'Hippone. Malgré son nom bien latin, Valerius était de langue grecque et s'exprimait en latin avec difficulté. Puis il sentait vieillir. Lors de son passage à Hippone, Augustin se trouvait un jour, un dimanche sans doute, à l'église au beau milieu des plebes. Valerius savait-il qu'Augustin était là? Toujours est-il que le vieil évêque ce jour-là a tenu devant l'assemblée des propos inhabituels. Il serait si bon, disait-il, d'avoir à ses côtés un aide, un prêtre de langue latine. Celui-ci s'occuperait mieux que lui-même du ministère de la parole auprès des croyants d'Hippone. Les bonnes gens d'Hippone ont compris tout de suite que leur hôte Augustin correspondrait parfaitement aux désirs de leur évêque et ont manifesté leur sentiment de façon musclée. Ainsi, dans les plus brefs délais, Augustin est devenu prêtre ${ }^{64}$.

Désormais il allait prêcher, même devant son évêque, même devant d'autres évêques ${ }^{65}$. Le vieux Valerius avait de l'audace, puis-

64. POSSIDIUS, Vita s. Aug. 4, 1-3: PL 32, 36-37; éd. M. PELLEGGINO, p. 50-52.

65. Je pense ici au De fide et symbolo que le prêtre Augustin prononça le 8 octobre 393 devant l'assemblée générale des évêques d'Afrique réunie a Hippone. 
qu'en Afrique cela était inédit ${ }^{66}$. Et il y avait plus. Augustin était moine et la vie monastique était alors regardée comme incompatible avec un sacerdoce réellement exercé au service d'une Eglise locale ${ }^{67}$. Mais Valerius faisait fi de cette incompatibilité. Il allait donner à Augustin une partie du jardin de son église afin d'y construire un monastère pour lui-même et quelques compagnons ${ }^{68}$. Sa rencontre avec Augustin lui a permis de montrer qu'il était une personnalité marquante et d'avoir un nom dans l'histoire du monachisme et du sacerdoce.

Augustin est devenu prêtre contre son gré. Tout en acceptant cette fonction, il a pleuré. Pourquoi? On risque de penser: parce qu'il allait perdre son "Thabor", sa chère vie monastique pure. Mais non, il s'agissait d'autre chose ${ }^{69}$. La véritable raison de ses larmes ne se trouvait pas là. Les gens d'Hippone se sont trompés à cet égard également, mais d'une tout autre façon. Possidius affirme en effet qu'ils attribuaient les larmes d'Augustin à un orgueil froissé et s'efforçaient de le consoler. Bien sûr, lui disaient-ils, il aurait mérité mieux que le sacerdoce, mais il devait comprendre que cette fonction était un premier pas vers l'épiscopat. Ces hommes bienveillants -ils ont parlé bono animo dira Augustin lui-même à leur propos- ${ }^{70}$ nous ont rendu un grand service: ils ont placé spontanément le sacerdoce et l'épiscopat dans les perspectives du carrié-

66. POSSIDIUS, Vita s. Aug. 5, 1-5: PL 32, 37-38; éd. M. PELLEGRINO, $52-54$.

67. Voir par exemple la Lettre 58, 5 de Jérôme a Paulin de Nole (PL 22, 582-583 ; CSEL 54,533-535), et le De Institutis coenobiorum XI, 18 de Jean Cassien (PL 49, 418; CSEL 17, 203).

68. Voir note 66 .

69. Je ne prétend nullement que cette perte l'aurait laissé indifférent. Bien au contraire. Dans son Saint Augustin et la fin de la culture antique, p. 360, note 1, H.- I. MARROU écrit a juste titre: "Tout au long de sa correspondance revient comme un leit-motiv la même plainte: "Je n'ai pas le temps, je suis débordé d'occupations". On sent quel fut le sacrifice que ce contemplatif, ce penseur, a consomme en acceptant la vie apostolique et les charges de l'action". A l'appui de cette remarque suivent une vingtaine de références, toutes tirées des Lettres de saint Augustin. Oui, celui-ci a éprouve cela comme un sacrifice, mais comme sacrifice librement et généreusement consenti. Dans sa Lettre XLVIII, 1 il écrit: “...nostras enim (orationes) saepe sauciat et debilitat caligo et tumultus saecularium actionum. Quas etsi nostras non habemus, eorum tamen qui nos angariant mille passus et iubemur ire cum eis alia duo, tanta nobis ingeruntur, ut uix respirare possimus credentes tamen, quod ille, in ciuis conspectu intrat gemitus compeditorum, perseuerantes nos in eo ministerio, in quo conlocare dignatus est cum promissa mercede, adiuuantibus orationibus uestris ab omni angustia liberabit". (PL 33, 188; CSEL 34, 2, 137). Augustin a bien su "quitter Dieu par Dieu". Dans sa tres importante lettre 21 il ne se plain précisément pas de la perte du "Thabor". Voir plus haut la fin de la note 4 à propos de l'étude de $A$. Wucherer-Huldenfeld.

70. Ep. 21, 2: PL 33, 88; CSEL 34, 50. 
risme: Augustin allait sans doute monter en grade. Cette réaction spontanée nous fait mieux comprendre celle d'Augustin lui-même.

Arrêtons-nous encore un instant à ce que nous raconte Possidius. Augustin, homme de Dieu, dit-il, tenait en réalité un raisonnement bien différent. S'il pleurait, c'était qu'il prévoyait les nombreux et grands dangers que le gouvernement et l'administration de l'Eglise allaient attirer sur sa vie, miulta et magna suae uitae pericula ${ }^{n}$. Mais quels dangers? Et quelle vie? Possidius ne le dit pas. Mais sa relation s'inspire nettement de la Lettre XXI d'Augustin à Valerius, écrite immédiatement après ces événements, et Augustin $\mathrm{y}$ est plus explicite que son biographe.

Cette Lettre entre aussitôt dans le coeur du sujet. Le sacerdoce est dangereux, estime Augustin. Dans la vie d'ici-bas, et surtout à l'époque actuelle —nous sommes en 391, à la fin de ce quatrième siècle dont le début a été marqué par Constantin, le premier empereur chrétien-, il n'y a rien de plus aisé, facilius, de plus réjouissant, laetius, et de plus agréable aux yeux des hommes, hominibus acceptabilius, que la fonction épiscopale, sacerdotale ou diaconale, si celle-ci est exercée dans un esprit superficiel, perfunctorie, et dans une ambiance de flatterie, adulatorie. Mais à la lumière de Dieu, il n'y a rien de plus malheureux, triste et honteux qu'une telle conception du ministère ${ }^{72}$. C'est précisément cette conception-là que les gens d'Hippone attribuaient à Augustin quand ils le voyaient pleurer et qui était sans doute leur conception personnelle de la fonction cléricale.

Augustin, quant à lui, ne pensait pas à une carrière ecclésiastique qui commençait mal en le mettant sur une voie de garage, mais aux dangers que le sacerdoce lui paraissait comporter. La Lettre XXI continue en effet: Mais dans la vie d'ici-bas, et surtout à l'époque actuelle, il n'y a en réalité rien de plus pénible, difficilius, de plus accablant, laboriosius, de plus périll e ux, periculosius, que ces fonctions. Mais' en même temps, devant Dieu, il n'y a rien de plus heureux, dès que le "soldat" accomplit son service dans l'esprit du Chef de l'armée. Mais quelle est la volonté du Chef? Dans les années précédentes de sa vie, Augustin ne l'avait pas appris. Il commençait tout juste à y voir plus clair quand la seconde place sur le navire lui a été confiée, à lui qui ne savait même pas se servir d'une rame ${ }^{73}$. Il suppose que Dieu l'a soumis à cette épreuve pour le punir de ses péchés. Et Augustin va s'expliquer à ce propos.

71. POSSIDIUS, Vita s. Aug. 4; voir note 64.

72. Ep. 21, 1: PL 33, 88; CSEL 34, 1, 49-50.

73. Ep. 21, 1: PL 33, 88; CSEL 34, 1, 50. 
Il avait connu un certain nombre d'évêques et de prêtres et bien souvent il les avait jugés sévèrement, s'estimant plus : savant et meilleur qu'eux, doctior et melior. Augustin avait donc fait' partie de ces intellectuels catholiques "anticléricaux" qui probaklement existeront aussi longtemps que l'Eglise qui, elle, a les promesses de la longévité. Maintenant, devenu prêtre lui-même, Augustin se rendait compte à quel point ses jugements avaient été téméraires. Mais il pouvait affirmer que déjà auparavant il avait compris que le ministère ecclésiastique est extrêmement d a n g e r e u x, periculosissimum. C'est précisément à cause de cela qu'il avait pleure quand il venait d'être ordonné prêtre ${ }^{74}$.

Dieu l'avait donc puni, mais il l'a fait par amour. Il a vouiu qu'Augustin connaisse sa maladie et en cherche la guérison. Il lui faut maintenant une santé qui soit en proportion avec les si d a ngereuses occupations, tam periculosa negotia, qui seront désormais les siennes. Il devra donc chercher avec soin les remèdes, tous les remèdes, contre sa maladie qui se trouvent renfermés dans les Ecritures. En priant et en lisant il doit faire de sorte qu'il trouve la santé nécessaire. Il avait précisément l'intention de se consacrer davantage à l'études des Ecritures quand il se voyait tout à coup jeté dans le ministère du sacrement et de la parole de Dieu. Que Valerius lui laisse donc encore un peu de temps libre, pour qu'il puisse rattraper son retard ${ }^{75}$. Ce retard ne concerne pas les grandes lignes de la révélation chrétienne. Il ose affirmer en effet qu'il est bien au courant de ce qui concerne le salut et qu'il s'y attache avec une foi totale. Mais le sacerdoce est une fonction publique et sociale. Il voudrait done savoir comment il faut mettre la conviction croyante qu'on a au service du salut des autres, sans rechercher son profit personnel mais celui du grand nombre, pour qu'ils soient sauvés ${ }^{76}$. Augustin pense trouver dans les Ecritures d'utiles conseils à cet égard, et il aimerait avoir du temps pour les découvrir, en demandant, en cherchant et en frappant, c'est-à-dire en priant, en lisant et en pleurant. D'utiles conseils scripturaires donc pour le gouvernement du peuple chrétien, bien qu'au secundus locus gubernaculorum pour l'instant. Mais ce n'était pas tout. Il désirait trouver aussi dans l'Ecriture comment se comporter au beau milieu des "injustes", inter manus iniquorum, comment soit y vivre avec une conscience bien robuste, soit y mourir afin de ne pas perdre da seu-

74. Ep. 21, 2: PL 33, 88; CSEL 34, 1, 50-51.

75. Ep. 21, 2: PL 33, 88; CSEL 34, 1, 50-51.

76. Ep. 21, 3: PL 33, 88-89; CSEL $34,1,51-52$. 
le vie qui compte aux yeux des Chrétiens humbles et tranquilles ${ }^{77}$. Somme toute; Augustin voulut trouver dans l'Ecriture des lumières sur deux aspects de ses occupations futures: dans quel esprit fallait-il gouverner?; quelle attitude fallait-il prendre par rapport aux "injustes"?

Avant d'avoir relu de près cette Letre XXI de saint Augustin, j'ai toujours pensé - et je n'étais pas seul à le faire-que saint Augustin a tout simplement demandé à Valerius quelques mois de répit afin d'approfondir ses connaissances bibliques. Mais cette impression trop globale n'est pas confirmée par une lecture précise de cette Lettre. Augustin se savait bien informé sur les grandes lignes de la révélation chrétienne. Ce qu'il désirait étudier était quelque chose de beaucoup plus précis. Il voulait des lumières sur l'exercice $\mathrm{du}$ sacerdoce, cette fonction publique et sociale. Au début de sa Lettre il avait déjà affirmé que le sacerdoce devient un malheur s'il est exercé dans une ambiance d'honneurs, de flatterie, de "bonheur" superficiel. Maintenant il dit qu'il faut l'exercer sans rechercher son profit personnel mais celui du grand nombre, afin qu'ils soient sauvés. La correspondance de cette phrase avec le début de la Lettre me paraît évidente. Il veut trouver dans la Bible des conseils d'ordre pratique, pours: s'occuper comme il faut de ses ouailles et des pécheurs.

Augustin demande donc à Valerius un délai, avant d'entrer effectivement en fonction. Jusqu'à Pâques! Et cela pour apprendre l' "agriculture" 78 , en lisant la Bible. Il voudrait que son absence -voulait-il rentrer à Thagaste pour quelque temps? - ne fût pas sans fruit pour l'Eglise du Christ et avantageuse pour ses "frères et co-serviteurs", fratrum et conseruorum ${ }^{79}$. La formule est précieuse et ajoute une précision sur l'idée qu'Augustin se faisait déjà à ce moment du sacerdoce: le prêtre est au service de ses frères, les fidèles, qui sont, comme lui-même et avec lui, des serviteurs de Dieu.

Mais soyons précis. Dans sa Lettre XXI saint Augustin nous disait textuellement qu'au moment où contre toute attente il allait être ordonné prêtre, il désirait déjà approfondir ses connaissances en matière biblique. Et qu'il l'a fait effectivement, c'est ce que démontrent ses réponses auy LXXXIII Questions. Cette collection couvre les périodes de Thagaste et d'Hippone jusqu'à la consécra-

77. Ep. 21, 3-4: PL 33, 89; CSEL 34, 1, 51-52.

78. Ep. 21, 5: PL 33, 89; CSEL 34, 1, 52-53.

79. Ep. 21, 6: PL 33, 90: CSEL 34, 1, 53-54. 
tion épiscopale de saint Augustin. Or la seconde partie de cet ouvrage manifeste un grand progrès en connaissance scripturaire, comparée avec la première partie ${ }^{80}$. Cependant -Augustin l'affirme lui-même, ce désir d'approfondissement était indépendant de son ordination sacerdotale et lui était antérieure. Le désir que son ordination inattendue a suscité chez lui concernait la découverte de conseils pratiques à propos de l'exercice concret du gouvernement de l'Eglise et du traitement des “"injustes". Ces deux désirs doivent être distingués soigneusement, plus soigneusement qu'on a l'habitude de le faire.

Date également de la période du sacerdoce d'Augustin la première Lettre qu'il ait écrite à Aurelius, évêque de Carthage et chef de l'épiscopat africain: c'est la Lettre XXII. Augustin y exprime d'abord sa désapprobation concernant les ripailles et les origies ${ }^{81}$ qui marquaient en Afrique le culte des morts, y compris celui des martyrs. Cette partie de la Lettre XXII ne nous occupera pas encore pour l'instant. Les querelles et les jalousies, écrit-il ensuite à Aurelius, sont malheureusement particulièrement fréquentes dans nos rangs, ceux du clergé, plus fréquentes que chez les laïques. Ces "maladies" trouvent leur source dans l'orgueil et dans l'avidité de louanges de la part des hommes. Le désir d'être loué pousse d'autre part à l'hypocrisie. Afin qu'on puisse résister à cette malsaine avidité de compliments, il faut absolument que la crainte et l'amour de Dieu nous soient inculqués par les abondants témoignages de l'Ecriture sainte. Il faut que les membres du clergé se montrent des exemples de patience et d'humilité, qu'ils acceptent, en matière de louanges, moins qu'on leur offre. De la part de ceux qui lui expriment leurs éloges, le prêtre ne doit accepter, ni le tout, ni rien. Et ce qu'il accepte dans le domaine des compliments et de l'honneur, il doit l'accepter, non pas pour lui-même -il doit se tenir devant Dieu avec une disponibilité totale et regarder les choses humaines comme négligeables-, mais pour le bien des fidèles. Mais d'autre part il doit comprendre qu'il ne peut faire rien de profitable pour eux s'il se diminue et s'abaisse trop ${ }^{82}$. C'est à cela que correspond

80. Voir l'introduction aux $L X X X I I I$ Questions par G. BARDY dans le vol. 10 des Oeuvres de saint Augustin. Paris, 1952.

81. Dans la composition de la lettre 22 saint Augustin s'inspire de Rom 13, 13-13: "...point de ripailles ni d'orgies, pas luxure ni de débauche, pas de querelles ni de jaolousies. Mais revêtez-vous du Seigneur Jésus Christ...". Qu'on se rapelle le rôle important que ce texte a joué dans la conversion de saint Augustin: Confessiones VIII, 12 (29).

82. "...si nimia deiectione uilescat". Comparer le Praeceptum, ligne 212 : ...dum nimia seruatur humilitas". 
d'un côté la parole: "Que personne ne méprise ton jeune âge!" ${ }^{83}$, mais aussi bien cette parole complémentaire, exprimée par le même saint Paul: "Si je voulais plaire à des hommes, je ne serais pas le serviteur du Christ" ${ }^{84}$. C'est une grande chose, continue-t-il, de ne pas se laisser impressionner par les honneurs et les éloges qui nous viennent de la part des hommes, ou bien et à plus forte raison, de supprimer toute vaine parade, et s'il faut bien retenir un peu de tout cela, de le faire servir intégralement au bien et au salut de ceux qui nous redent cet hommage. Ce n'est pas pour rien qu'il est dit dans le Psaume 52,6: "Dieu brisera les ossements de ceux qui veulent plaire à des hommes" ${ }^{85}$. Y a-t-il en effet quelque chose de plus mou, de plus dépourvu de fermeté et de force (d' "ossements") qu'un homme qui se laisse paralyser par ce que disent les mauvaises langues, tout en sachant que ce qu'ils disent est faux? La douleur éprouvée à cause de ces propos ne déchirerait pas les entrailles de l'âme si l'amour des éloges n'avait pas brisé ses ossements ${ }^{86}$.

Dans sa Lettre XXI à Valerius, saint Augustin avait exprimé son désir de trouver dans l'Ecriture de bons conseils concernant l'exercice $d u$ sacerdoce. N'est-il pas intéressant de rencontrer dans la Lettre XXII quelques échantillons de ces recommandations?

En lisant la suite de sa Lettre à Aurelius on constate que le tout premier à qui saint Augustin parle dans ce contexte est lui-même en personne. Quant à Aurelius, il lui dit: Je me sens assuré en ce qui concerne la force intérieure de votre personne. Aussi les paroles que j'échange avec vous, les dis-je à moi-même. Mais je suis sûr que vous voudrez bien considérer avec moi combien tout cela est grave, combien tout cela est difficile. Car nul ne se rend compte des forces de cet ennemi tant qu'on ne lui a pas déclaré la guerre. On arrive à se passer d'éloges si les autres refusent d'en octroyer, mais il est difficile de ne pas s'en réjouir quand on nous en présente. Et pourtant, l'attachement de notre esprit là-haut à Dieu ${ }^{87}$ devrait être si grand que nous soyons capables de rappeler à l'ordre ceux qui nous louent indûment -à condition que nous puissions les rap-

83. $1 \operatorname{Tim} 4,12$.

84. Gal 1, 10.

85. "Deus confringet ossa hominibus placere volentium". Il y a probablement une allusion à ce texte dans le Praeceptum, lignes 99-100: "Illi (sc. deo) ergo uir sanctus (voir note 25) timeat disciplicere, ne uelit feminae male place$r e "$. Si ce rapport n'a pas été rmarqué jusqu'ici, c'est sant doute à cause du lilbellé inhabituel du verset. Dans le Psautier romain en effet le Ps. 52, 6 porte: "Deus dissipat ossa hominum sibi placentium".

86. Ep. 22, 7-8: PL 33, 92-93; CSEL 34, 1, 59-60.

87. “...suspensio in deum". Comparer le Praeceptum, ligne 4: “...sit anima una et cor unum in deum". 
peler à l'ordre!-, pour éviter qu'ils nous attribuent des qualités que nous n'avons pas, ou qu'ils attribuent à nous des qualités qui nous viennent uniquement de Dieu, ou qu'ils louent des qualités que nous avons réellement, même peut-être en abondance, mais qui ne sont nullement louables, comme par exemple toutes celles que nous avons en commun avec les animaux, ou avec les impies. $\mathbf{S i}$, au contraire, on nous loue conformément à la réalité mais à cause de Dieu, félicitons alors ces gens à qui plaît le véritable bien, mais ne nous félicitons pas nous-mêmes du fait de plaire aux hommes ${ }^{88}$. Félicitons-nous au contraire si nous sommes réellement, devant le regard de Dieu, ce que ces gens nous pensent être, et si les qualités qu'ils louent en nous conforméent à la réalité, ne sont pas attribuées à nous mais à ce Dieu qui nous donne tout. Voilà, dit Augustin, ce que je me répète tous les jours. Ou plutôt, c'est ce que Dieu lui-même répète tous les jours. C'est lui qui me donne ses préceptes salutaires, qu'ils se trouvent dans les saintes Ecritures, ou qu'ils se présentent intérieurement à l'âme. Mais cela n'empêche pas, ainsi conclut Augustin, que dans mon violent combat avec l'Adversaire, je recoive souvent des blessures: je n'arrive pas toujours à éloigner de moi le plaisir causé par la présentation d'un compliment ${ }^{89}$.

Je ne crois pas me tromper en pensant que ce long passage est un intéressant complément à la Lettre qu'Augustin avait adressée à son évêque valerius au lendamain de son ordination sacerdotale. Il voulait lire la Bible, disait-il à Valerius, pour savoir dans quel esprit il devait exercer le gouvernement de l'Eglise. La Lettre à Aurelius nous rêvèle ce qu'il y a trouvé et quelles conclusions il en a tirées.

Mais il avait aussi voulu trouver dans la Bible de bons conseils concernant l'attitude qu'il devait prendre à l'égard des "injustes". A ce propos également la lecture de la Lettre XXII est des plus instructives. N'est-elle pas tout entière un combat contre les ribailles et les orgies lors du culte des morts, et contre les querelles et les jalousies si fréquentes dans les rangs du clergé?

Vici ce que saint Augustin a lu dans l'Epitre aux Romains -c'est le texte même qui a joué le rôle qu'on sait dans sa propre

88. Voir la note 85 .

89. Ep. 22, 8: PL 33, 93; CSEL 34, 1, 60-61. Saint Augustin s'en prend à l'épiscopaltrie" dans son Sermon Morin Guelf, 32, 4: "Et uides homines nihil loqui aliud. Vidisti episcopum? Salutatis episcopus? Unde uenis? Ab episcopo. Quo pergis? Ad episcopum.... Simul audiamus, simul in schola condiscipuli ab uno magistro Christo discamus, cuius cathedra ideo est in caelo, quia prius crux fuit in terra": $M A \mathrm{I}, 566$; PLS 2, 63 . 
conversion-: "Comme il sied en plein jour, conduisons-nous avec dignité: point de ripailles ni d'orgies, pas de luxure ni de débauche, pas de querelles ni de jalousies. Mais revêtez-vous du Seigneur Jèsus-Christ et ne vous souciez pas de la chair pour en satisfaire les convoitises" ${ }^{90}$. Augustin fait remarquer à Aurelius que l'Eglise est très sévère quand il s'agit de luxui'e et de débauche. Mais pourquoi le serait-elle uniquement dans ce domaine? N'est-ce de trois catégories de péchés que l'Apôtre parle aux Romains? Ciest pourquoi Augustin s'étend longuement sur les orgies dans la première partie de sa Lettre et sur les querelles et les jalousies dans la seconde. Il espère pouvoir lutter contre ces abus par la collaboration de tous les chefs de l'Eglise africaine, sous la conduite de l'évêque de Carthage, Aurelius ${ }^{91}$. Mais dans quel esprit doit être mené ce combat? Augustin a lu chez saint Paul: "Frères, même dans le cas où quelqu'un serait pris en faute, vous les spirituels, rétablissez-le en esprit de douceur et de bienveillance" ${ }^{92}$. On ne doit donc pas essayer d'éliminer ces ripailles d'une façon rugueuse, aspere, ni avec dureté, duriter, ni d'une manière autoritaire, modo imperioso, mais en informant plutôt qu'en commandant, magis docendo quam iubendo, en exhortant plutôt qu'en menaçant, magis monendo quam minando. C'est-là la conduite à prendre avec la multitude. La sévérité, elle, est plutôt à employer danş des cas individuels. Et s'il arrive aux dirigeants de l'Eglise d'exprimer des menaces, il faut que cela se fasse avec douleur, qu'on fonde ses avertissements sur l'Ecriture sainte et sur les sanctions qui y sont prédites. C'est Dieu luimême que les pécheurs doivent craindre à travers les paroles des chefs de l'Eglise. Il ne faut pas qu'ils aient peur des dirigeants, à cause de leur puissance. Ainsi on touchera d'abord les spirituales, ceux qui sont perméables à l'action du Saint-Esprit, ou ceux qui sont proches des spirituales. C'est par leur influence, auctoritas, et par leurs avertissements, à la fois très calmes et très pressants, lenissimis et instantissimis, que les autres, le grand nombre, devront être touchés ${ }^{33}$.

Voilà ce que saint Augustin a découvert en étudiant l'Ecriture à propos de l'attitude à prendre par le clergé vis-à-vis des "injustes".

Nous avons déjà rencontré la Lettre XLVIII qu'Augustin : a écrit à Eudoxius et les autres moines de Capraria. Que devait faire

90. Voir note 81 .

91. Ep. 22, 3-4: PL 33, 9192; CSEL 34, 1, 56-58.

92. Gal 6, 1

93. $E p .22,5$ : PL 33, 92; CSEL 34, 1, 58. 
un moine si l'Eglise lui demandait d'assumer une charge pastorale dans un diocèse? Augustin ne voulait pas, nous l'avons vu, qu'il préfère alors sa tranquillité aux besoins de l'Eglise. Mais ce qui nous intéresse maintenant est moins la pensée d'Augustin à propos de $l$ 'otium que ce qu'il pensait du negotium pastoral dont ce moine allait éventuellement être chargé.

Augustin explique à Eudoxius et ses frères que le ministère pastoral est une chose dangereuse. Qu'on ne se précipite donc pas pour devenir prêtre ou évêque. Ils ne devaient pas assumer ces charges avec une arrogance impatiente, elatione auida, mais avec bonté et humilité, miti corde et cum mansuetudine. Qư'on se tienne loin de la paresse, desidia, mais aussi de cette fierté orgueilleuse, superbia, qui est le grand danger de la vie cléricale. On risque de s'y dresser sur ses ergots, extolli, d'être réduit à rien et de se fondre totalement dans une vantardise qui veut se faire valoir, mais qui fait en réalité qu'on se dissipe en fumée et étincelles ${ }^{94}$.

Dans le dix-neuvième livre de la Cité de Dieu, -nous l'avons vu également-, saint Augustin compare aussi la vie sans charge pastorale, la uita otiosa, avec la vie dans le ministère, uita actuosa. La première ne doit pas être oisive à tel point qu'on n'y pense pas au prochainn, mais la seconde ne doit pas être si active qu'elle en oublie la quête de la contemplation de Dieu. Dans la première il ne faut pas chercher son plaisir dans une oiveté inerte, mais dans l'autre -et ici nous arrivons à ce qui nous intéresse dans cette partie de notre recherche - il ne faut pas aimer les honneurs terrestres, ni la puissance que la uita actuosa implique: il s'agit du salut des autres, du bien de ceux que l'on gouverne. Dans le gouvernement, on ne doit pas aimer le fait de praeesse, mais celui de prodesse ${ }^{95}$, pas celui d'être à la tête d'autres personnes, mais celui de pouvoir leur être utile ${ }^{96}$.

Ce passage de la Cité de Dieu et celui de la Lettre XLVIII que nous avons lu avant lui, font abstraction du combat contre le Mal, l'autre aspect de l'exercice du ministère pastoral, pour insister uniquement sur celui du praeesse. Mais dans d'autres textes saint $\mathrm{Au}-$ gustin souligne plus particulièrement combien la tâche du pasteur est redoutable quand des brebis s'égarent. Ainsi dans le Sermon 46 qui porte précisément le nom de $D e$ pastoribus.

94. Ep. 48, 2: PL 33, 188; CSEL 34, 2, 138.

95. L'antithèse "être à la tête", "être utile" se trouve également dans $E p$. 134, 2; Serm. 46, 2; Serm. 340, 1: Serm. Morin Guelf, 32, 1; Contra Faustum $\mathrm{XX}, 56$.

96. De Civitate Dei XIX, 19: PL 41, 647-648; CC 48, 686-687. 
Dans ce Sermon saint Augustin commente longuement Ezéchiel 34, 1-16 dont voici les versets 2-4: "Pasteurs, ainsi parle le Seigneur Yahvé. Malheur aux pasteurs d'Israel qui se paissent eux-mêmes. Les pasteurs ne doivent-ils pas paître le troupeau? Vous vous êtes nourris de lait, vous vous êtes vêtus de laine, vous avez sacrifié les brebis les plus grasses, mais vous n'avez pas fait paître le troupeau. Vous n'avez pas fortifié les brebis chétives, soigné celle qui était malade, pansé celle qui était blessée. Vous n'avez pas ramené celle qui s'égarait, cherché celle qui était perdue. Mais vous les avez régies avec violence et dureté".

D'abord quelques mots brefs sur le début du Sermon.

A qui faut-il penser, se demande le prédicateur, quand on lit: "Malheur aux pasteurs qui se paissent eux-mêmes"? Il s'agit de ceux dont l'Apôtre dit: "Tous recherchent leurs propres intérêts, non ceux de Jésus Christ" "97.

Et Augustin s'explique: Nous qui nous trouvons à cet endroit élevé ${ }^{98}$, symbole de notre haute fonction, nous avons deux qualités, l'une celle d'être des Chrétiens, l'autre celle d'être des chefs, des praepositi. Le fait d'être des Chrétiens nous a été donné pour nousmêmes, mais celui d'être des chefs a été pour vous. Dans le premier ce qui a été envisagé est notre profit à nous, dans le second c'est uniquement le vôtre. Il y a un grand nombre de simples Chrétiens - "simples" dans le sens qu'ils ne sont pas des chefs- qui parviennent à Dieu après un voyage plus facile sans doute, avec une démarche je pense d'autant moins encombrée qu'ils portent un fardeau moins lourd. Mai quant à nous, outre la qualité de Chrétiens qui nous obligera à rendre compte à Dieu de notre propre vie, nous avons aussi la qualité de chefs, ce qui nous obligera à rendre compte à Dieu de la façon dont nous avons accompli notre tâche. Si je vous fais part de ce grave souci, c'est pour que vous ayez de la compassion pour nous et que vous priez pour nous. Car le jour viendra où tout sera amené en jugement ${ }^{99}$. Si ce jour est peut-être encore éloigné pour les hommes en général, chacun d'eux est bien près du dernier jour de sa propre vie... ${ }^{100}$.

97. Fil 2, 21.

98. L'idée de locus superior se trouve également dans $E p$. 166, 2, les Enarrationes in Ps. 66, 10 et 126, 3, Sermones 91, 5; 101, 4; 137, 13-14; 355, 2: Morin Guilf, 32, 8, et De Civitate Dei XIX, 19. Elle se trouve aussi dans le Praeceptum, lignes 234-235: "(Praepositus) inter uos, quanto in loco superiore, tanto in periculo maiore uersatur".

99. Qoh 12, 14.

100. Serm. 46, 2: PL 38, 271; CC 41, 529-530. 
Les chefs ont été établis afin de veiller aux intérêts de ceux qu'ils gouvernent. C'est pourquoi quiconque exerce sa fonction pastorales de telle façon qu'il trouve du plaisir dans le fait d'être chef, qu'il y cherche des témoignages de considération et d'estime et qu'il a uniquement en vue ses propres avantages, se paît soi-même et non les brebis ${ }^{101}$. Les mauvais pasteurs chez Ezéchiel se sont nourris du lait des brebis. Cela veut dire qu'ils exerçient leur fonction pastorale en vue de biens matériels. Ils se sont vêtus de la laine des brebis. Autrement dit, ils s'accaparaient avidement les honneurs qui étaient dûs à leur charge, et donc à Dieu. Les honneurs sont en effet comme des vêtements, qui dissimulent tant de choses. Ils couvrent les infirmités du pasteur, si bien qu'on en parle comme s'il était un ange... ${ }^{102}$.

Ce terme d" "ange" est suggéré à saint Augustin par saint Paul qui dit dans la Lettre aux Galates: “...vous m'avez accueilli comme un ange de Dieu, comme le Christ Jésus... Je vous rends ce témoignage: s'il avait été possible, vous seriez arrachés les yeux pour me les donner" ${ }^{103}$. Puit saint Paul ajoute Let c'est avec cette citation que commence dans le commentaire de saint Augustin le dévelonpement qui nous intéresse maintenant particulièrement-: "Alors, suis-je devenu votre ennemi en vous disant la vérité?" ${ }^{104}$.

Saint Augustin met cette phrase de saint Paul en rapport avec les paroles du Seigneur qui disent que le bon pasteur doit fortifier les brebis chétives, soigner les brebis malades, panser les brebis blessées, ramener celle qui s'est égarée, chercher celle qui est perdue. Le pasteur doit donc penser au bien-être des brebis, même au risque de leur déplaire. Etre pasteur, ce n'est pas seulement être éventuellement aux petits soins de quelques bonnes gens, ni le fait d'être dangereusement l'objet d'éloges, d'approbations, de sympathie, voire de flatteries; c'est aussi être l'objet de critiques, de contestations et de haine quand on fait son devoir en disant la vérité; et courir de graves risques en se taisant ${ }^{105}$.

Evitons, poursuit saint Augustin, de tenir aux fidèles le langage que voici: Vivez comme vous le voudrez, soyez sans inquiétude, Dieu ne perdra personne; conservez seulement la foi chrétien-

101. Serm. 46, 2: PL 38, 271; CC 41, 530.

102. Serm. 46, 5-6: PL 38, 272-273; CC 41, 532-533.

103. Gal 4, 14-15.

104. Gal 4, 16.

105. Serm. 46, 20 : PL 38, 281; CC 41,546, Comparer le Praeceptum, lignes 114-115: "Magis quippe innocentes non estis, si fratres uestros, quos indicando corrigere potestis, tacendo perire permittitis". 
ne, Dieu ne perdra pas ceux qu'il a rachetés au prix de son sang. Si vous voulez vous distraire en allant aux spectacles; allez-y; quel mal y a-t-il? Et ces festivités qu'on célèbre à travers des villes entières avec de joyeux festins où l'on se régale ensemble à des tables publiques et où le gens se sentent heureux —c'est unerillusion, car en réalité ils s'y perdent-, allez-y, célébrez-les; la miséricorde de Dieu est grande, elle pardonne tout. "Couronnez-vous de roses avant qu'elles ne fanent", dit le proverbe. Faites à votre guise des festins dans la maison même de votre Dieu ${ }^{106}$, gorgez-vous avec les vôtres de nourriture et de vin; ces choses ont été crées et données pour cela, pour que vous en jouissiez; car Dieu ne les a pas données aux impies et aux païens, pour vous les refuser à yous. Bien sûr, isì nous vous parlions ainsi, nous attierons peut-être des assemblées plus nombreuses. Il est vrai que certains estimeraient peut être en nous écoutant que nous ne sommes pas sains desprit, mais! il ne s'agirait là que du petit nombre et nous aurions pour nous laigrande foule. Mais tout cela serait paitre nous-mêmes, au lieu de paitre les brebis ${ }^{107}$.

Parmi les brebis on trouve quelques exemplaires qui sont forts et gras, c'est-à-dire qui, en se nourrissant eux-mêmes de la verritế sont solides et fermes et font un bon usage des paturages que la faveur de Dieu leur offre. Mais que font les mâuals pasteurs? ${ }^{2}$ est trop peu pour eux de négliger les brebis languissantes et inflirmes, celles qui s'égarent et se perdent; non, il faut encore quautant qu'ils le peuvent, ils tuent ces brebis fortes et grasses. Mais, me demandera-ton, comment leur donnent-ils la mort? C'est par leur mauvais exemple. Serait-ce en vain qu'un serviteur de Dieu, mis à une place éminente parmi les brebis du Pasteư suprême, a reçu cette recommandation: "A l'égard de tous, offre en ta personne un exemple de bonne conduite" ${ }^{109}$, et: "Montre-toi un modèle pour les croyants" ${ }^{10}$. En effet, il arrive souvent qu'une brebis même vigoureuse, qui regarde la mauvaise conduite de son pasteur, de son praepositus, et qui détourne alors ses regards des préceptes du Seigneur,

106. Dans le Breviarium Hipponense se lit, sur le numéro 29 , la décision suivante: "Ut nulli episcopi uel clerici in ecclesia conuiuentur, nisi forte transeuntes hospitiorum necessitate illic reficiant, populi etiam ab huiusmodi conuiuiis, quantum potest fieri, prohibeantur": éd. C. "MUNIER, CC 4, 149, 41.

107. Serm. 46, 8: PL 38, 274; CC 41, 534-535.

108. Voir plus loin note 154 .

109. Tit 2, 7. La même citation a une place importante dans $E p .32,7$ (voir note 86) et Sermo 355, 1. Voir aussi le Praeceptum, lignes 227-228: “Circa omnes seipsum bonorum praebeat exemplum".

110. 1 Tim 4, 12. 
se mette à tenir ce langage: Si mon praepositus vit ainsi, qui suisje pour agir autrement? Voilà de quelle façon le mauvais pasteur donne la mort à la brebis forte. Elle vit encore, il est vrai, mais ce pasteur est un assassin quand même. Que dire alors quand il s'agit de brebis infirmes? ${ }^{111}$.

Et puis que faut-il penser de ces pasteurs qui ne préparent pas leurs ouailles aux épreuves qui les attendent nécessairement dans cette vie, mais qui vont même jusqu'à leur promettre un bonheur terrestre, un bonheur que Dieu n'a pas promis aux siècles qui se dếroulent ici-bas? Pourquoi formulent-ils de telles promesses? C'est par peur de choquer ceux à qui ils parlent. Pourquoi leur dire ceci: Si ut vis bien dans le Christ, avec piéte, tu auras tout ce qui est bon en abondance; si tu n'as pas d'enfants, ta femme t'en donnera, et tu les élèvera tous, sans en perdre aucun. Est-ce là, mauvais pasteur, l'édifice que tu construis? Tu construis une maison sur le sable. Dieu n'a rien promis de pareil. Quant à toi, ne cesse pas de garder devant tes yeux ce Dieu qui reprend et encourage, qui effraie et console, qui donne des coups et apporte la guérison. Le bon pasteur doit prendre soin de ses brebis même robustes, et surtout il doit soigner les brebis malades, panser les brebis blessées, ramener celle qui s'est égarée, chercher celle qui est perdue ${ }^{112}$.

Bien que saint Augustin, dans son Sermon 46 de pastoribus, ne manque pas de signaler le danger des éloges que la charge pastorale entraîne, il y est surtout préocupé par les devoirs redoutables que le pasteur a dans le cadre de la protection de ses brebis contre l'illusion des faux "biens" et contre les forces du mal.

Cette préoccupation, principale dans ce Sermon, devient unique dans la Lettre XCV, adressée à Paulin de Nole.

Augustin s'y déclare bien angoissé: Quelle crainte, quel effroi dans ce domaine, mon Paulin, saint homme de Dieu, quelles ténèbres! On dirait qu'il s'agit de cela dans le Psaume qui dit: "Crainte et tremblement me pénètrent, un frisson m'étreint. Et je dis: Qui me donnera des ailes comme à la colombe, que je m'envole et me pose? Voici, je m'enfuirais au loin, je giterais au désert" ${ }^{113}$. Mais c'est peut-être au désert que cet homme a éprouvé ce qu'il fait suivre: "J'attendais quelqu'un pour me sauver de l'abattement et de la tempête". Tant il est vrai ${ }^{114}$ que la vie de l'homme sur la terre est éprouvante ${ }^{115}$.

111. Serm. 46, 9: PL 38, $274 ;$ CC 41, 535.

112. Serm. 46, 11-12: PL 38, 275-277; CC 41, 537539.

113. Ps 54, 6-9. 
Sévir, ne pas sévir, quel embarras! Dans les deux cas en effet nous voulons que notre attitude contribue au salut, soit de ceux dont nous estimons la punition nécessaire, soit de ceux que nous traitons avec indulgence. Mais il est difficile de connaittre la mesure à garder dans le premier cas. Car il faut considérer non pas seulement la nature et le nombre des actes coupables, mais aussi les forces spirituelles des uns et des autres, ce que chacun peut supporter, à quoi il risque de se montrer revêche. Nous devons en effet éviter deux choses: d'un côté qu'il ne marque le pas, mais aussi et surtout qu'il n'en arrive à renoncer à la marche. Tout cela est un puits obscur et sans fond. Je ne sais même pas dans quelle mesure la crainte d'une sanction imposée par un homme est réellement bénéfique. Le nombre de ceux qu'une telle sanction a rendus pires est peut-être plus grand que le nombre de ceux qu'elle a corrigés. Combien il est accablant de penser que souvent, en punissant un coupable, on le fait périr, mais qu'en le laissant impuni, on en fait périr un autre ${ }^{116}$.

Et pour bien montrer combien ces soucis lui étaient personnels et qu'en tout cela il parlait de nouveau surtout à lui-même, Augustin ajoute: Dans ces choses-là, je pèche tous les jours ${ }^{117}$.

Bien sûr, il sait qu'il peut trouver à ce propos de bons conseils dans la Parole de Dieu -ne s'est-il pas mis à la lire dans cette optique su lendemain de son ordination sacerdotale?-, mais il n'est pas facile de savoir dans quelles circonstances il faut appliquer l'une ou l'autre de deux sentences qui, en soi, semblent bien contradictoires. Dans la première Lettre à Timothée saint Paul écrit en 5, 20: "Les coupables, reprends-les devant tout le monde, coram omnibus ${ }^{118}$, afin que les autres en éprouvent de la crainte" mais le Seigneur dit lui-même en Matthieu 18,15: "Si ton frère vient à pécher, reprends-le seul à seul, inter te et ipsum solum" "11. Dans 1a première Lettre aux Corinthiens saint Paul dit en 4,5: "Ne portez pas de jugement prématuré, afin de ne pas être l'objet d'un jugement vous-mêmes". La première fois, le terme de "jugement" est précisé par "prématuré"; pourquoi ne l'est-il pas la seconde fois aussi? Prenons ensuite la Lettre aux Romains en 14,4: "Toi, qui estu pour juger un serviteur d'autrui? Qu'il reste debout ou qu'il tom-

114. Job 7,1 .

115. Ep. 95, 3: PL 33, 353; CSEL 34, 1, 509.

116. Ep. 95, 3: PL 33, 353; CSEL 34, 1, 508. Comparer le Praeceptum, lignes 128-129: "...de uestra societate proiciatur. Non enim et hoc fit crudeliter, sed misericorditer, ne contagione pestifera plurimos perdat".

117. Ep. 95, 3: PL 33, 353; CSEL 34, 1, 508 . 
be, cela ne concerne que son maître; d'ailleurs il restera debout, car le Seigneur a la force de le soutenir". Il est évident que l'Apôtre panle ici de ceux "du dedans", c'est-à-dire d'autres Chrétiens. Mais contrairement à cela il ordonne de juger ceux du dedans en écrivant aux Corinthiens en I, 5, 12-13: "Qu'ai-je à faire de juger ceux du dehors? N'est-ce pas ceux du dedans que vous avez à juger? Enlevez le pervers du milieu de vous!" Combien de soucis et de tremblements dès qu'il faut passer aux actes dans ce domaine! Ecrivant encore aux Corinthiens; en II, 2,7, saint Paul les exhorte à la mesure dans les châtiments, de peur que le malheureux coupable ne vienne à sombrer dan une trop grande tristesse. Mais qu'elle est terrible, la précision qu'll ajoute dans le même contexte: "Il s'agit de n'être pas dupes dé 'Satan: nous n'ignorons pas ses desseins" 120 :

Arrêtons-nous une derniere fois pour faire le point:

1. Agustin a bien compris, comme Jean Chrysostome, que la vie monastique pure, sans charge pastoràle, risque d'être vécui égoïstement. 2. Il estimait néanmoin' que les moines-laïques he devaient nullement se précipiter pour assumer un ministère pastoral: il fallait que l'Eglise les y invite, mais dans ce cas ils ne devaient pas refuser de se mettre à sa disposition. 3 úne fois mis à la tête d'une Eglise locale, l'ancien moine-laïque devait faire connaître et aimer la vie des moines-lailques. 4. Cette vie, "contemplative", n'est pas une iners uacatio. "Je dors, mais mon coeur veille", disait l'Epouse du Cantique Lamour de la Vérité pousse à une activité intense, la sanctification personnelle et la quête de la connaissance savoureuse des "choses de Dieu" 5. Mais c'est également dans un autre sens que la vie monastique pure n'est pas une iners uacatio. La "sagesse" n'est pas une propriété privée, à garder pour soi égoïstement, mais à communiquer à autrui, aux "frères" de la même communauté ou à ceux du dehors. 6. Les moines-laïques appelés par l'Eglise au ministère pastoral n'échappent nullement au risque de vivre égoïstement. Bien au contraire. Ils ont à redouter un double et grave danger. D'abord le danger de trouver leur bonheur dans le praeesse et non pas dans le prodesse, dans le fait d'occuper la première

118. Il y a allusion à 1 Tim 5, 20, dans la Règle de saint Augustin; voir le Praeceptum, lignes 123-125: "Si autem negauerit, tunc nescienti adhibendi sunt alii, ut iam coram omnibus possit, non ab uno teste argui, sed a duobuis uel tribus conuinci".

119. Comparer le Praeceptum, ligne 122: “...ne forte possit, secretius correptus, non innotescere ceteris".

120. Ep. 95, 3: PL 33, 353; CSEL 34, 1, 508-509. 
place et non pas dans celui d'être au service du bien des autres. Il faudrait dire: le bien des autres pécheurs. C'est ici que se présente l'autre danger du ministère pastoral: tout pécheur qu'il est, le pasteur doit punir les coupables, ni trop, ni trop peu, en tenant compte de toutes les circonstances. Dans les deux domaines, Augustin a avoué ses propres faiblesses, une fois en ce qui concerne le premier danger, dans la Lettre XXII à Aurelius; une fois en ce qui concerne le second, dans la Lettre XCV à Paulin de Nole. Vraiment, il savait pourquoi il ne fallait pas se précipiter pour devenir prêtre ou évêque.

Dans sa Lettre XXI, écrite à son évêque Valerius au lendemain de son ordination sacerdotale, Augustin avoue qu'il avait été une sorte d'intellectuel catholique "anticlérical". Mais il ajoute que déjà dans cette période de sa vie, il avait compris combien il était dangereux d'assumer des responsabilités publiques dans l'Eglise. Or il semble que cette conviction plonge ses racines dans la formation philosophique de saint Augustin. Grâce à elle il savait depuis longtemps qu'il est périlleux d'être à la tête d'autres hommes.

Dans une publication antérieure ${ }^{121}$ j'ai eu l'occasion d'attirer l'attention des lecteurs sur un passage important du livre II du De Oräine où se manifeste précisément la pensée philosophique à laquelle je viens de faire allusion. A l'intention de ses jeunes disciples Licentius et Trygetius, Augustin y trace une règle de vie qui, d'après le contexte, remonterait à Pythagore. Il serait mieux de dire qu'elle remonte plus globalement à des conceptions éthiques courantes de l'Antiquité et que le nom de Pythagore est à placer entre guillements. Or, chose très intéressante, dans ce résumé d'éthique ancienne, les deux soucis dont nous avons longuement parlé à propos du sacerdoce et de l'épiscopat de saint Augustin se manifestent déjà. Rappelons-nous qu'à l'époque saint Augustin n'était même pas baptisé et qu'il ne pouvait nullement soupçonner qu'il serait plus tard prêtre et évêque. A propos du mal à combattre chez autrui et à propos de l'exercice de l'autorité Augustin formule à l'intention de Licentius et Trygetius les préceptes suivants: A l'égard des fautes des leurs, qu'ils évitent absolument toute colère ou qu'ils la refrènent à tel point qu'elle semble chassée. Qư'ils ne haïssent personne. Qu'il n'y ait pas de vice auquel ils ne s'efforcent de remédier. Qu'ils fassent grande attention à éviter la prodigalité dans la punition, l'avarice dans le pardon. Qu'ils ne punissent que pour améliorer;

121. Voir mon article "Par les praecepta uiuendi à la spiritalis pulchritudo. "Pithagore", le De Ordine de sant Augustin et sa Règle": Augustiniana 22 (1972 469-510. 
qu'ils évitent toute indulgence favorisant le mal... Dans les fautes d'autrui, qu'ils ne soient pas importuns lorsqu'il s'agit d'un méfait involontaire ${ }^{122}$.

Voilà pour la lutte contre le mal chez autrui. Il est intéressant de remarquer qu'il s'agit de méfaits commis éventuellement par les sui de Licentius et Trygetius, c'est-à-dire par leur futur entourage immédiat, composé de sujets. Toutes ces règles visent à la formation de "patrons", patres familias et hommes politiques à la fois.

Ensuite l'exercice de l'autorité en général: Qu'ils regardent comme les leurs - suos, de nouveau ce terme, mais avec une nuance plus prononcée d'intimité- tous ceux sur qui pouvoir leur a été donné. Qu'ils soient à leur service, comme s'ils rougissaient de les commander; qu'ils commandent comme s'ils faisaient leur joie de les servir ${ }^{123}$.

Toutes ces recommandation n'ont rien d'original. Il s'agit de la fine fleur de l'étique "politique" classique ${ }^{124}$.

Le lecteur a sans doute reconnu dans ces phrases du De Ordine les germes de tout ce que dira saint Augustin plus tard sur l'exercice de l'autorité sacerdotale et épiscopale. Mais il faut souligner que cette correspondance frappante a une conséquence très importante. Quand saint Augustin parlera des charges dangereuses du sacerdoce et de l'épiscopat, il fera en effet totalement abstraction de leurs éventuels aspects "charismatiques". Pour lui le sacerdoce et l'épiscopat comportent de graves dangers parce qu'il s'agit de cas d'espèce de l'exercice de l'autorité. L'exercice de l'autorité entraîne à ses yeux toujours des risques, qu'il s'agisse de l'autorité d'un père de famille, d'un homme politique ou d'un évêque. Que l'évêque se trouve aussi dangereusement nanti de certains pouvoirs "divins", sacramentaux par exemple, cela n'entre pas dans les perspectives augustiniennes.

Nous trouvons une confirmation de ce que je viens de dire dans le Praeceptum, la Règle de saint Augustin. Le Praeceptum suppose dans le monastère dont il ordonne la vie la présence de deux autorités, le praepositus et le presbyter. Le praepositus est le moine-laïque s'occupe de la direction "quotidienne" de sa communauté. Le rôle du presbyter, le prêtre de la maison, est à la fois plus affacé et plus important que celui du praepositus. Il doit se manifester quand il faut réagir contre des fautes particulièrement graves com-

122. De Ordine II, 8, 25: PL 32, 1006; CC 29, 121.

123. De Ordine II, 8,25: PL 32,1006; CC 29,121.

124. Voir l'article cité dans note 121 . 
mises par un membre du groupe et aussi, d'après certains textes parallèles, quand il s'agit de questions de doctrine et d'exégèse ${ }^{185}$. Ce prêtre est nommé quatre fois dans le Praeceptum. Or il est extrêmement intéressant intéressant de constater que les réflexions de saint Augustin sur les dangers inhérents au sacerdoce et à l'épiscopat ne trouvent aucun écho dans ces quatre passages sur le prêtre de la maison. En revanche ce que saint Augustin dit sur le moine-laïque qui exerce l'autorité quotidienne dans cette communauté, est peut-être le meilleur résumé qui existe de tout ce que nous avons lu plus haut sur l'exercice du sacerdoce et de l'épiscopat. C'est tout simplement parce que saint Augustin regarde le prêtre et l'évêque comme les praepositi de leur troupeau, et non pas comme des "nantis de charismes".

Voici ce que dit le Praeceptum à propos du praepositus du monastère: Il lui appartient de veiller à la pratique de ces préceptes, de ne rien laisser enfreindre par négligence, mais de redresser et de corriger ce qui n'aurait pas été observé... Qu'il ne place pas son bonheur dans l'asservissement des autres sous son autorité ${ }^{126}$, mais dans les services ${ }^{127}$ qu'il leur rend par charité. Par l'honneur, devant vous, qu'il soit à votre tête; par la crainte, devant Dieu, qu'il se tienne à vos pieds ${ }^{128}$. Qu'il soit pour tous un modèle de bonnes oeuvres, s'appliquant à corriger les instables, à ranimer ceux qui manquent de courage, à soulever les faibles et à exercer la patience envers tous ${ }^{129}$. Qu'il observe ces règles de bon coeur, qu'il en impose le respect. Et, quoique les deux soient nécessaires, il cherchera à gagner votre affection plutôt qu'à susciter votre crainte ${ }^{130}$, toujours pensant au compte ${ }^{131}$ qu'il devra rendre de vous à Dieu. C'est pourquoi en obéissant mieux, vous ne faites pas seulement preuve de compassion envers vous-mêmes, mais aussi envers lui ${ }^{132}$ : il se trou-

125. Voir mon article "Praepositus ad presbyterum referat quod modum uel uires eius excedit": Augustiniana 27 (1977).

126. Textes parallèles dans De Ordine II, 8, 25; Confessiones X, 4, 6; Enarrationes in Ps. 1, 1; De Civitate Dei XV, 7; XIX, 12-16.

127. Textes parallèles dans De Sermone Domini in monte II, 19, 65; Ep. 23, 1; Enarrationes in Ps. 99, 11; Sermo Morin Guelf, 32, 3; De opere monachorum 29, 37; De Civitate Dei XIX, 14-16; Contra ep. Parmeniani III, 2, 16.

128. Textes parallèles dans Enarrationes in Ps. 66, 10; 126, $3 ;$ Serm. 146, 1.

129. Tit 2, 7. Saint Augustin cite le même texte dans Ep. 22, 7; Quaestiones Heptat. 119 ; Serm. 46, 9; 269,$4 ; 355,1$.

130. Textes parallèles dans Confessiones X, 36, 58-59; Ep. 22, 9, 31; Enarrationes in Ps. 116, 3.

131. Textes parallèles dans Ep. 29, 7; Enarrationes in Ps. 50, 24; 126. 3; Serm. 46, 2; 82, 15; 91, 5; 137, 15; 146, 1; 339, 1 (ou S. Frangip. 2, 1); 340, 1. 132. Textes parallèles dans Ep. 26, 6; 29, 7; 137, 1; Serm. 232, 8; De opere monachorum 33 (41). 
ve parmi vous, en effet, $\grave{a}$ une place d’autant plus dangereuse qu'elle est plus élevée ${ }^{133}$.

A une place plus dangereuse, in periculo maiore... C'est le titre même de cette recherche. Rappelons-nous que ce terme de periculum est revenu maintes fois au cours de ces pages et qu'il se trouvait déjà dans le récit que Possidius donnait de l'ordination sacerdotale de saint Augustin. C'est ce periculum qu'Augustin redoutait dans le sacerdoce, beaucoup plus que la perte de son "Thabor".

Je pense que ce passage du Praeceptum constitue un excellent résumé de nos lectures antérieures. Mais je pense aussi que tous ces textes, de Possidius et d'Augustin, constituent un excellent commentaire des lignes du Praeceptum que nous venons de lire.

Ia. Saint Augustin: obéissance et direction spirituelle.

Un excursus

Il me semble que les pages précédentes jettent aussi quelque lumière sur les réflexions de saint Augustin en matière de direction spirituelle et d'obéissance.

On sait quel rôle important la direction spirituelle a joué chez les Pères du désert. Parmi eux, il y avait des sages, des gens d'une sainteté notoire, ayant une exceptionnelle expérience de la vie spirituelle, et qui avaient en même temps le don d'être des guides pour les autres, spécialement pour les derniers venus. Ils transmettaient d'âge en âge la grande tradition monastique. C'étaient les "Anciens" appelés aussi "Apas" ou Pères. Ils donnaient une doctrine de sainteté, fondée surtout sur la Bible, illustrée par des anecdotes tirés de la vie des premiers Pères du désert.

Une bonne idée du genre d'enseignement que donnalent les "Apas" à leurs flls spirituels nous est donnée par les Collationes de Jean Cassien ${ }^{134}$. Ces Collationes se présentent précisément comme des comptes rendus d'instructions par des "Anciens". On y voit Cassien et son ami Germain assis aux pieds de l'Apa Pinuphius, de l'Apa Nesteros, de l'Apa Isaac... Cassien et Germain posent à chacun des questions d'intérêt spirituel et l'interrogé répond en donnant un long exposé sur la prière, par exemple, ou sur le poison mortel qu'est la colère, ou sur l'avarice qui trahit une carence dans le renocement au monde accompli jadis par tel ou tel moine. Ces

133. Praeceptum, lignes 220-232. A propos de "la place plus élévée et par conséquent plus dangereuse" voir la note 98.

134. PL 49, 477-1328; CSEL 13. 
Conférences n'ont rien d'académique. Il s'agit beaucoup moins de donner une définition de l'avarice, par exemple, que d'expliquer ses effets néfastes, et de montrer comment le moine doit éviter ou éliminer ce malheur.

Ceci dit, on comprendra mieux ce que dit saint Jérôme dans un discours sur l'Obéissance ${ }^{135}$, donné par lui à ses frères du monastère de Bethléem. Il s'agit dans ce discours beaucoup plus de réceptivité par rapport à la direction spirituelle que d'obéissance au sens courant de ce terme. Pour saint Jérôme la vertu monastique par excellence est l'obéissance. Rien, dit-il, ne plaît tant à Dieu que cette attitude... Il n'appartient pas aux fils de juger leurs pères (au pluriel, remarquons-le). Personne ne va chez un maitre pour faire la classe à son maître. Le maître lui dit: "Tu es venu chez moi pour que je t'apprenne les lettres; si je te fais un modèle en te disant: Ecris cette lettre comme moi", alors tu dois imiter le maitre que tu t'es choisi. Une fois qu'on aura appris ce qui était à apprendre, on va chez un maître plus compétent. Pourquoi dire tout cela? Pour que nous nous montrions obéissants envers nos pères. Point de déférence envers ses pères, point de déference envers Dieu... Qui méprise les pères méprise le Christ qui est dans les pères. Je dis cela parce que ici, pour nous, dans le monastère, la principale et même la seule vertu est l'obéissance... A elle seule, l'obéissance vaut mieux que toutes les autres vertus... Si l'on n'obéit pas, c'est qu'on se croit meilleur que celui auquel on n'obeit pas ${ }^{136}$.

Ce n'est pas sans raison qu'avant de lire ces paroles de saint Jérôme, j'ai fait quelques remarques élémentaires sur la direction spirituelle chez les moines de cette époque. Entendant parler d'obéissance, nous pensons spontanément à l'exécution d'un ordre pratique donné par quelqu'un qui commande dans n'importe quel genre de societé, que ce soit l'ordre d'un chef de bataillon ou l'ordre d'un supérieur religieux. Sans exclure ces perspectives, le texte de saint Jérôme parle essentiellement d'autre chose. Rappelons-nous: les pères sont au pluriel; ces pères sont des maître qui enseignent; les élèves doivent imiter le modèle que les maîtres présentent; ils choisissent leur maître et, après quelque temps, ils vont chez un maître plus compétent; si l'on ne veut pas obéir, ce n'est pas qu'on s'estime juridiquement indépendant, mais qu'on se croit meilleur que le maître. Dans tout cela il ne s'agit pas essentiellement de l'exécution d'ordres donnés, mais de la disponibilité pour recevoir la

135. CC 78, 552-558.

136. CC 78,552 . 
direction des pères dont - comme le dit Cassien- la vie a été louable et bien exercée dans leur jeunesse monastique et qui se sont réglés sur les saines traditions de leurs aînés ${ }^{137}$.

Quant à Cassien lui-même, les Apas qu'il a rencontrés ne lui ont pas seulement octroyé leur direction, ils lui ont parlé aussi de cette attitude par excellence que la direction spirituelle suppose chez les "fils spirituels", 1' "obéissance". Nous devons nous asujettir, a-t-il appris, au joug de l'obéissance, dans la simplicité de notre coeur et sans aucun déguisement, en sorte qu'il n'y ait plus aucune autre volonté en nous que celle de nostre Apa. Mais cela ne peut s'accomplir que par celui qui se regarde non seulement comme mort au monde, mais encore comme un fou et un insensé, accomplissant sans discuter tout ce que les "anciens" lui disent de faire, considérant cela comme un oracle de Dieu et comme une loi sainte et inviolable ${ }^{138}$. Le vrai discernement s'acquiert uniquement par l'abandon au jugement des "anciens" non seulement de tout ce que l'on doit faire, mais aussi de ses pensées, en sorte que, ne se fiant nullement en son jugement personnel, on acquiesce toujours aux décisions des "anciens" et que l'on reçoive d'eux la connaissance de ce qu'il faut juger comme bon ou mauvais ${ }^{139}$.

Il n'est pas facile de décrire correctement la position de saint Augustin dans ce domaine. Ou plutôt, il est absolument nécessaire d'entrer dans son monde spirituel par la bonne porte afin de trouver les bonnes perspectives. Il y a une différence nette entre ce qui prédomine chez Augustin et ce que nous venons de lire chez Jérôme et Cassien. Chez eux, et ailleurs, on voit les maitres spirituels en train d'inculquer l'attitude qu'il faut à ceux qui sont appelés à se laisser guider; mais Augustin (qui parle de préférence à lui-même) se préoccupe plutôt d' inculquer l'attitude qu'il faut à ceux qui sont appeler à commander, à diriger, à enseigner: ils ne doivent jamais faire écran à Dieu. Cette façon d'aborder les problèmes de l'obêissance et de la direction spirituelle est si inattendue, semble-t-il, que même des hommes aussi compétents que Gustave Bardy ont fait fausse route à ce propos. On le verra dans un instant.

Il ne faudrait pas croire cependant que la préoccupation principale des Jérôme et des Cassien ait été totalement étrangère à Au-

137. Conl. II, 13: PL 49, 543; CSEL 13, 53.

138. De Institutis coenobiorum XII, 32, 2: PL 49, 475; CSEL 17, 230.

139. Conl. II, 10; PL 49, 537; CSEL 13, 48. Voir l'Appendice IV: L'enfantvieillard, dans Grégoire de Nysse, Traité de la virginité (Sources Chrétiennes 119) par M. AUBINEAU, 575-577. 
gustin. Il est vrai qu'en parlant d'obéissance Augustin pense généralement à l'attitude de l'homme vis-à-vis de Dieu ${ }^{140}$. Il reste que pour lui l'obéissance d'un homme à un autre homme est une partie intégrante de toute société humaine, qu'elle soit monastique ou non. Partout où il y a une sociétté humaine, il y a forcément des gens qui commandent. Sans cela il n'y aurait pas d'unité, pas de cohérence et par conséquent pas de société. Même dans ces quasisociétés que sont les bandes de brigands, les compères exécutent des orores, ceux du "patron", et la désobéissance s'y paye même plus chez qu'ailleurs. Mais cette fausse imitation de sociétés plus respectables ne manque pas de souligner la nécessité d'une autorité dans n'importe quelle société et d'une attitude obéissante par rapport aux dirigeants ${ }^{141}$. Bien entendu, aux yeux d'Augustin les dirigeants sont là pour la société, et non pas la société pour les dirigeants. Dans la Règle d'Augustin le praepositus du monastère est le serviteur des serviteurs de Dieu. Mais cela n'empêche nullement que le même Praeceptum demande aux frères de ce monastère d'obéir à leur praepositus comme, dans un foyer, on doit obéir au père de famille ${ }^{142}$. Saint Augustin parle relativement peu de l'obéissance que les hommes doivent à d'autres hommes, mais il en parle d'une façon bien nette. Mais retournons à Gustave Bardy dont le nom a été cité un peu plus haut.

Dans le très important et souvent si intéressant Dictionnaire de Spiritualité, le long article sur la Direction spirituelle est en réalité une suite de plusieurs articles. La partie qui, entre autres, traite de saint Augustin est de la main de Gustave Bardy. Elle porte le titre que voici: "La direction spirituelle en Occident jusqu'au 11" siècle" "143. Après une brève introduction, son auteur traite des trois grands maîtres de l'époque patristique, Ambroise, Jérôme (surtout de lui, bien entendu) et Augustin. Voici les premières des lignes que Bardy a consacrées à ce dernier:

"La vie de saint Augustin a été trop absorbée par le ministère épiscopal pour qu'il ait eu le temps de se consacrer au service d'âmes choisies et de les conduire vers la perfection. Il serait injuste de ne pas rappeler son nom, car il ne s'est pas contenté de rechercher la sainteté pour lui-même; il s'est toujours efforcé de la

140. Voir A. MANRIQUE, "Concepto monástico de obediencia en san Agustín": Revista Agustiniana de Espiritualidad 2 (1961) 18-40 et "Obediencia agustiniana y voluntad de Dios": 6 (1965) 177-184.

141. De Civitate Dei XIX, 12: PL 41, 638; CC 48, 676.

142. Praeceptum, ligne 217: "Praeposito tamquam patri oboediatur...".

143. Volume III, 1061-1083. 
montrer comme le but à atteindre, non pas à tout le peuple fidèle, ce qui est impossible, mais à des groupes de clercs, de moines, de religieuses, voire à des hommes ou à des femmes vivant dans le monde" ${ }^{144}$.

Etrange formule qui semble bien trahir un embarras. Dans la seconde phrase, la maịn gauche de Bardy reprend intégralement ce que, dans la première, sa main droite avait repoussé. Et quant au fait qu'Augustin n'aurait pas fait de la direction spirituelle faute de temps, c'est un argument qui ne pourra satisfaire aucun familier de saint Augustin. Que n'a-t-il pas fait, sans en avoir le temps! On a l'impression qu'à propos de la direction spirituelle Gustave Bardy se soit vaguement aperçu de quelque chose d'inhabituel, sans qu'il ait su cerner le problème. Il n'a pas compris qu'Augustin s'intéressait dans ce domaine surtout aux bonnes dispositions du "maître".

Parmi les textes d'Augustin qui selon Bardy ont un intérêt pour l'histoire de la direction spirituelle en Occident se trouve la Lettre CCLXVI, adressée à Florentine, une jeune "vierge consacrée" qui vivait avec ses parents. Le début du billet nous apprend que la mère de la jeune fille avait récemment envoyé une lettre à Augustin. Florentine, avait écrit sa maman, serait très désireuse de lui poser quelques questions. Mais elle n'osait pas faire le premier pas. Il faudrait que l'évêque lui-même ouvre l'entretien et donne à Florentine la permission de parler ${ }^{145}$.

Je fais, écrit saint Augustin, ce que tu désires, bien que ce soit une autre que toi qui m'ait exprimé ce désir. Je ne veux pas avoir l'air de te laisser grossièrement devant la porte quand ta confiance vient $\mathrm{y}$ frapper; c'est maintenant à toi de parler, si tu crois avoir quelque chose à me demander ${ }^{146}$.

L'évêque pense-t-il recevoir de Florentine des questions concernant sa vie personnelle intérieure et sa perfection chrétienne? Il s'agissait dans son idée certainement de questions d'intérêt religieux, mais pour le reste aucune spécification n'est prévue. La suite de la Lettre parlera de questions sur des "choses divines", des diui$n a$, dans un cadre de pietas et de uera sapientia ${ }^{147}$.

144. Oeuvre citée, 1065.

145. Ep. 256, 1; PL 33, 1089; CSEL 57, 647.

146. Ep. 256, 1: PL 33, 1089; CSEL 57, 647.

147. Ep. 256, 3: PL 33, 1090 et 1091 ; CSEL 57,649 et 650 . Quant à la pietas, voir mon article "Ecouter la parole de Dieu sans interrompe ni protester": Augustiniana, 27 (1977) p. 142. 
Mais represons la lecture de la Lettre:

Cependant, Florentine, n'espère pas avec trop de certitude trouver auprès de moi la réponse à tout ce que tu pourrais me demander. Et si ton attente venait à être trompée, ne dis pas: Augustin a été plus hardi que prudent en me permettant de lui poser mes questions. Je ne me suis pas proposé comme un docteur accompli, mais comme un homme qui doit progresser avec ceux qu'il est appelé à éclairer... Assurément, dans les choses mêmes que je sais tant bien que mal, j'aime mieux te trouver instruite que constater que tu as besoin de mes connaissances. Car nous ne devons pas souhaiter que d'autres soient ignorants afin d'avoir à enseigner ce que nous savons: il vaut beaucoup mieux que nous soyons tous des disciples de Dieu. C' est ce qui se réalisera à la perfection dans la patrie d'en haut... L'Ecriture nous fait prendre garde en disant: "Que tout homme soit prompt à écouter, mais lent à parler" 148. Le psalmiste du Psaume 50 a compris que ceux qui écoutent gardent l'humilité avec facilité, cette humilité qui est si difficile pour ceux qui instruisent ${ }^{149}$. Il faut bien qu'un maître occupe un lieu plus élevé, mais à ce niveau il est malaisé de se défendre effectivement contre les attaques de l'orgueil ${ }^{150}$.

C'est-là une pensée que nous avons déjà rencontrée dans le Traité 57 sur l'Evangile selon saint Jean ${ }^{151}$. La Lettre à Florentine s'inscrit nettement dans les mêmes perspectives que les autres textes que nous avons parcourus.

Augustin continue: Ne vois-tu pas quel danger nous courons, nous de qui on attend non seulement que nous soyons des docteurs, mais encore que nous enseignions les choses divines, les diuina, nous qui ne sommes que des hommes? Toutefois, dans ces travaux et ces périls, il y a une consolation, unique: celle de voir que les autres parviennent au point de ne plus avoir besoin d'être enseignés par un homme, qui que ce soit... Le Seigneur lui-même, admirable médecin de l'enflure de l'âme dont je parlais plus haut, n'a-t-il pas dit: "Ne cherchez pas à être appelé Maître par les hommes, parce que vous n'avez qu'un seul Maître, le Christ"?... ${ }^{152}$ Sache donc que ma joie à cause de ta foi, ton espérance et ta charité sera d'autant

148. Jue 1, 19.

149. En disant en 10: "Auditui meo dabis exultationem et laetitiam et exultabunt ossa humiliata".

150. Ep. 256, 2: PL 33, 1090; CSEL 57, 648-649. Quant a superiorem locum, voir note 98 .

151. Voir notes 33,34 et 35 . 
plus sûre, d'autant plus solide et d'autant plus éclatante de santé ${ }^{153}$ spirituelle que tu auras moins besoin pour t'instruire, non seulement de moi, mais d'absolument aucun homme. Cependant, tes bons parents m'ont demandé de ne pas te refuser mon modeste secours, operula mea, dans les choses où tu pourrais avoir besoin d'être instruite par moi... J'attends dons les questions que tu voudras me poser... ${ }^{154}$.

Lisons maintenant la magnifique fin de cette Lettre:

Tiens pour absolument certain que même quand tu pourras apprendre quelque chose par mon intermédiaire et d'une manière salutaire, ton Maître véritable sera toujours le Maître intérieur de l'homme intérieur. C'est lui qui te fait reconnaître au plus profond de toi-même, la vérité de ce que l'on vous dit. Car celui qui plante n'est rien, ni celui qui arrose, mais tout vient de Dieu qui donne l'accroissement ${ }^{155}$.

"Vous n'avez qu'un seul Maître, le Christ". Thème important dans cette Lettre, texte fréquemment cité par saint Augustin. Il est sans doute remarquable que cette citation ne se trouve pas une seule fois dans l'oeuvre monastique de Cassien ${ }^{156}$. Celui-ci pensait en effet plutôt aux bonnes dispositions des disciples qu'a celles des maîtres.

La Lettre de saint Augustin à Florentine est un véritable trésor. Nulle part ailleurs il ne dit d'une façon aussi émouvante, aussi personnelle, que les chef̣s et les maîtres sont d'humbles serviteurs

152. $M t ~ 23,8-10$.

153. Tanto sanius. Dans ce contexte sanus a un rapport évident avec la "vertu théologique" de la dilectio. De Serm. Denis 19, 6 (voir plus loin note 168) précise catégoriquement: "Hoc est in membris Christi caritas, quod est in membris corporis sanitas". Je pense que cette idée explique perfaitement le sens orationes saniores dans le Praeceptum, lignes 198-201: "Si autem inuicem se laeserunt, inuicem sibi debita relaxare debebunt, propter orationes uestras, quas utique, quanto crebriores habetis, tanto saniores habere debetis". Il s'agit d'une récitation charitable du Notre-Père, avant tout pendant la célébration, quotidienne a Hippone de l'Eucharistie.

154. Ep. 256, 3-4: PL 33, 1090-1091; CSEL 57, 649-650.

155. 256, 4: PL 33, 1091; CSEL 57, 650.

156. Il a pourtant une allusion à ce texte dans Conl. II, 15. Jean Cassien y souligne que Saul de Tarse a été renvoyé par Jésus-Christ au senior Ananie. Le Christ aurait pu l'enseigner lui-même. S'il ne pas fait, c'est precisément de peur que ce qui eut été juste dans le cas de l'Apôtre ne fut pour l'avenir un mauvais exmple qui encourageat la presomption, et que chacun ne se persuadat ne devoir, comme l'Apôtre, reconnaître pour maître et docteur que Dieu seul, plutôt que de se former a l'école des anciens. La différence entre Cassien et Augustin dans ce domaine est ici particulièrement nette. 
qui ne doivent pas refuser leur modeste secours, leur operula. Mais ils risquent toujours d'interposer orguelleusenment un écran entre Dieu et les membres de son peuple. L'idée du $d$ ange $\mathrm{r}$ est revenue dans cetee Lettre: Ne vois-tu pas, Florentine, quel danger nous courons...? ${ }^{157}$.

\section{Anima unica Christi}

La deuxième partie de la contribution de M. Jean-Marie Leroux au Colloque de Chantilly traitait des "principes de foi déterminant la vie monastique". C'est là que la communication de M. Leroux et la mienne se touchaient de près. Pour Chrysostome comme pour Augustin la vie monastique ne trouve pas sa finalité en elle-même: il s'agit de la cause de Dieu. Jean Chrysostome a estimé qu'il ne faut pas y chercher une perfection hypothétique dans des vertus, mais reproduire autant que faire se peut l'image du Verbe incarné ${ }^{158}$. Augustin, lui, ne peut pas penser au Verbe incarné sans penser en même temps au Christus totus. Ce qu'il faut rechercher dans le cadre du "Christ total", c'est d'avoir l'anima unica Christi, qui est aussi l' "âme une de l'Eglise". Exprimé d'une façon plus individuelle ou d'une façon plus collective, l'idéal monastique des deux grands serviteurs de Dieu est fondamentalement le même. Ni pour l'un, ni pour l'autre, la vie monastique ne saurait être la voie vers une vie éternelle meilleure que celle des autres hommes. Le Christ est venu appeler tous les pécheurs, c'est lui l'Alpha et l'Oméga.

Dans le chapitre précédent nous avons cité à plusieurs reprises la très intéressante et très riche Lettre XLVIII de saint Augustin. Mais j'ai gardé la lecture de son début pour maintenant, étant donné que ses premières lignes donnent une magnifique illustration de ce que saint Augustin comprenait par l'anima unica Christi.

Eudoxius et ses moines, auxquels la Lettre XLVIII a été adressée, avaient demandé à saint Augustin si un moine doit, ou ne doit pas, quitter la vie monastique pure, sans charges pastorales, pour assumer la direction d'une Eglise locale. Ils avaient eu la bonne idée de s'adresser à quelqu'un qui pouvait parler en connaissance de cause puisque saint Augustin était lui-même un moine devenu prêtre, puis évêque, d'Hippone.

157. Voir note 154.

158. Article cité dans la note 3, 130-131 
Voici le début de sa réponse:

Quand nous pensons à la tranquillité dont vous jouissez dans le Christ, nous aussi, mes frères et moi, nous trouvons du repos malgré les labeurs pénibles de toute sorte qui marquent notre existence. Nous trouvons du repos en vous, nos chers frères dans le Seigneur. Car nous sommes un seul corps sous une seule Tête, si bien que d'une part vous êtes en nous actifs dans le ministère, tandis que de l'autre nous vivons en vous la vie monastique pure. Il est dit en effet: "Un membre souffre-t-il?, tous les membres souffrent avec lui. Un membre est-il à l'honneur?, tous les membres prennent part à sa joie". C'est pourquoi nous vous exhortons, nous vous prions, nous vous conjurons, par la si sublime humilité du Christ et par sa si compatissante majesté, de vous souvenir de nous dans vos saintes prières que nous croyons être bien plus rigoureusement attentives que les nôtres. Car nos prières sont souvent déchirées et affaiblies à cause des ténèbres et du vacarme de nos activités dans un domaine si peu religieux ${ }^{159}$ :

Augustin qui - nous l'avons déjà vu- n'allait nullement demander à Eudoxius et ses frères de se précipiter dans l'activité pastorale, a néanmoins voulu qu'ils aient un esprit ecclésial. L'anima unica Christi a une universalité qui s'oppose à tout repli sur soimême.

\section{Le col de la tunique}

Nous avons pu être assez bref sur la deuxième partie de l'exposé de M. Leroux. Sur son troisième point, également d'ordre théologique, nous aurons à nous étendre plus longuement.

Si le moine est en tout premier lieu un Chrétien comme tous les autes membres du Corps $d u$ Christ, avec une vocation finale identique à celle des autres, quel est alors son rôle spécifique? Chrysostome pense que tout Chrétien doit être signe du salut, proclamant, par sa vie surtout, que le salut est déjà réalisé dans sa plénitude, que le Christ est présent parmi nous et que nous vivons déjà une vie nouvelle ${ }^{160}$. Mais Chrysostome estime que le moine est ce signe d'une façon particulière. C'est dans un certain isolement, dans l'organisation d'un mode de vie spécifique, que le signe mo-

159. Ep. 48, 1: PL 33, 187-188; CSEL 34, 2, 137.

160. Article cité dans la note 3,136 . 
nastique fonctionne, et cela au milieu du monde, afin d'en être le ferment. D'une part le ferment doit s'incorporer à la pâte, mais en même temps il doit conserver son caractère spécifique, précisément pour faire lever la pâte. "Les monastères sont des phares qui brillent de haut pour éclairer de loin ceux qui viennent à eux; établis dans le port, ils invitent tout le monde à partager leur tranquillité, ne permettant pas que ceux qui les voient fassent naufrage ou demeurent dans les ténèbres" ${ }^{161}$.

Dans l'oeuvre de saint Augustin un texte très intéressant nous permet de voir dans quel sens l'évêque d'Hippone diff̣ère ici de l'évêque de Constantinople. Il s'agit de son commentaire du Psaume 132 (133).

Voici le début de ce Psaume:

1. "Voyez: qu'il est bon, qu'il est doux d'habiter en frères tous ensemble.

2. C'est une huile excellente sur la tête, qui descend sur la barbe, qui descend sur la barbe d'Aaron, sur le col de sa tunique".

L'huile excellente est ce mélange d'huile d'olive, de myrrhe, de cinnamome, de canne et de casse qui, au jour du sacre du grandprêtre, était versé abondamment sur la tête du pontife. Autrement dit, c'est le chrême, le chrisma, pontifical.

Aux yeux de saint Augustin, Aaron, comme chaque grand-prêtre, est l'image du Christ. Et le Christ est le Christus totus, la Tête et le Corps. C'est que le saint chrême est descendu de la Tête sur la tunique. Le Grand-prêtre de la nouvelle Alliance est lui-même la victime offerte au Père céleste. Il a été crucifié et mis au tombeau, pour ressusciter le troisième jour et monter au ciel. C'est de là que l'Esprit Saint a été envoyé, que le chrême de l'onction sacerdotales est descendu sur la tunique ${ }^{162}$ qui représente le Corps ecclésial du Christ ${ }^{163}$.

Le chrême a inondé tout d'abord la barbe, symbole de ces gens vigoureux, entreprenants et dynamiques qui, les premiers, avaient à subir les assauts des persécutions. Saint Augustin pense ici aux Apôtres, aux premiers Chrétiens de Jérusalen et, parmi eux, à Etienne, le protomartyr. Leur courage n'a pas été brisé, ni, surtout,

161. Article cité dans la note $3,136$.

162. Enarrationes in Ps. 132, 7: PL 37, 1733; CC 40, 1931.

163. Enarrationes in Ps. 132, 9: PL 37, 1734; CC 40, 1932. 
leur charité unifiante. Par ses paroles Etienne admonestait courageusement ceux qui le persécutaient, mais son coeur était tout rempli d'amour pour eux et il demandait au Seigneur de ne pas leur imputer le meurtre qu'ils étaient en train d'accomplir ${ }^{164}$.

Pois le chrême est descendu plus bas, de la barbe du Grandprête sur le col de sa tunique. Le col ou le bord de la tunique est cette partie très ornementée de la robe par laquelle passe la tête. Si le vêtement ne comportait pas ce col, le Grand-prêtre ne pourrait pas revêtir sa tunique. Le col est donc la partie de la robe qui l' "achève" en tant que robe, qui fait que le vêtement est vraiment un vêtement prêt à être porté. Sans cela, il ne s'agirait encore que d'un morceau de drap.

Augustin a déjà dit que la qualité essentielle de l'Eglise est son unité dans la charité. Pour cette raison le col de la robe ecclésiale, l'élément qui l'achève, est l'ensemble de tous ceux qui, tels les frères du Psaume 132, savent habiter en frères tous ensemble, qui savent porter les fardeaux les uns des autres et accomplir ainsi le grand commandement du Christ. Le Christ peut se mettre la tunique ecclésiale grâce à la concorde fraternelle ${ }^{165}$.

Or la concorde fraternelle est précisément la raison d'être des communautés cénobitiques. C'est pour constituer une seul âme et un seul coeur que les cénobites se rassemblent ${ }^{166}$. En étant ce qu'ils sont appelés à être les moines cénobites apportent une contribution importante au fonctionnement cie l'Eglise en tant que fraternité dans le Christ ${ }^{167}$.

Je pense qu'un des "Sermons Denis" nous aidera à bien saisir ici la pensée de saint Augustin. Il y dit:

Dans le corps humain il y a des fonctions diverses à accomplir, mais la santé du corps, elle, est une. Or dans les membres du Corps du Christ la charité est ce que dans les membres du corps physique est la santé... La santé, qui est commune à tous les membres de ce corps, est plus précieuse que les fonctions spécifiques que chaque membre accomplit. L'un est un oeil, l'autre n'est qu'un extremus digitus, mais celui qui contribue le plus au fonctionnement harmonieux du tout y est le plus bénéfique pour la santé du tout. En revanche, tout ce qui dans un corps est malade cause des ennuis au reste du corps. Tous les membres saints du corps contribuient à la

164. Enarrationes in Ps. 132, 7: PL 37, 1733-1734; CC 40, 1931-1932.

165. Enarrationes in Ps. 132, 9: PL 37, 1734; CC 40, 1933.

166. Enarrationes in Ps. 132, 6: PL 37, 1733; CC 40, 1931.

167. Enarrationes in Ps: 132, 9: PL 37, 1734-1735; CC 40, 1933. 
guérison de ce qui est malade et bien souvent la santé de ce membre malade se rétablit grâce à la santé de l'ensemble. Mais si une gangraine inguérissable s'est installée dans ce membre malade, il est bon pour les autres que ce membre soit enlevé de l'organisme par une opération ${ }^{168}$.

Pour Jean Chrysostome les monastères sont des phares qui illuminent la nuit du monde, les moines sont des signes par excellence du salut. Pour Augustin les monastères sont plutôt appelés à être des principes de santé pour l'Eglise. Je pense que la présentation d'Augustin est la plus redoutable. La signe demande à être vu, mais risque de tromper en donnant de la réalité une apparence flatteuse. Le principe de santé, lui, doit avant tout fonctionner. Que l'Eglise accomplisse vraiment sa vocation d'être une fraternité en JésusChrist, cela dépend pour une bonne part de ce que les moines cénobites font de l'anima unica Christi, eux qui se sont rassemblés pour avoir une seule âme et un seul coeur tendus vers Dieu.

A l'époque où nous vivons il est particulièrement intéressant de noter qu'aux yeux de saint Augustin cette concorde fraternelle avait une dimension d'ordre social. Cel aspect de ses conceptions n'a d'ailleurs pas échappé à l'historiographie marxiste ${ }^{169}$. Dans les monastères dont Augustin nous parle les "frères" venaient d'horizons socialement bien divers. Sa Règle évoque la présence d'anciens riches et d'anciens pauvres ${ }^{170}$, tandis que son De opere monachorum oppose plus concrètement ceux qui avaient été des senatores et des praediorum domini à ceux qui avaient été des opifices et des rustici ${ }^{171}$. Tout cela fait penser à une société où régnait une profonde inégalité sociale. Afin de favoriser leur unanimité et leur concorde tous ces gens vivaient désormais dans une pauvreté absolue, c'est-à-dirle sans aucune posiselssion personnelle. Pour les anciens riches l'entrée au monastère représentait un grand sacrifice, mais aussi un grand danger. L'orgueil humain se glissant partout ils ris-

168. Sermo Denis 19, 6: PL 46, 891-892; MA T, 103-104. Voir note 153. Comparer le Praeceptum, lignes 204-206: "Qui autem numquam uult petere uenian, aut non ex animo petit, sine causa est in monasterio, etiam si inde non proiciatur".

169. Voir surtout H. J. DIESNER, Der Untergang der römischen Herrschaft in Nordafrika (Weimar 1964) 128-130.

170. Praeceptum, lignes 65-67: “...ne contingat detestanda peruersitas, ut in monasterio, ubi, quantum possunt, fiunt diuites laboriosi, fiant pauperes delicati".

171. De opere monachorum 25 (33): "Nullo modo enim decet, ut in ea uita, ubi senatores fiunt laboriosi, ibi fiant opifices otiosi, et quo ueniunt relictis deliciis suis qui fuerant praediorum domini, ibi sint rustici delicati”: PL 40, 573; CSEL 41, 580. 
quaient de continuer à tirer gloire du lustre de leur famille fortunée, de se vanter parce qu'ils faisaient vivre la communauté, d'avoir du dédain pour les autres qui avaient été pauvres ou qui avaient même vécu dans une pauvreté extrême ${ }^{172}$. Mais ces derniers n'étaient nullement à l'abri de certains piéges. Ils risquaient de chercher dans le monastère avant tout une promotion sociale, un bienêtre qu'ils n'avaient pas connu auparavant, la fréquentation quotidienne de gens dont ils n'osaient même pas s'approcher quand ils étaient encore dans "le monde" 173. L'unanimité et la concorde qui sont le fondement de la vie monastique augustinienne demandent à tous de se respecter mutuellement d'une façon libre et humble à la fois. Les anciens pauvres doivent honorer les anciens riches, non pas à cause de l'argent qu'ils ont possédé, mais à cause de leur dignité humaine et chrétienne. Les anciens riches ne doivent plus dédaigner les pauvres, mais les honorer: ne sont-ils pas, eux-aussi, des "temples de Dieu", des frères devant le Seigneur? ${ }^{174}$.

L'ancienne inégalité qui n'existe plus, n'a pas manqué de provoquer des contrecoups, bien réels eux, au niveau des santés. Les anciens riches avaient été gâtés et n'étaient pas tous capables de supporter le régime peu raffiné du monastère. D'autre part certains anciens pauvres avaient vécu dans une pauvreté telle que leur santé en était ébranlée. L'unanimité et la concorde qui devaient régner entre les frères du monastère leur demandaient de tenir généreu-

172. Praeceptum, lignes $5-12 ; 23-29 ; 143-144$ : "Et non dicatis aliquid proprium, sed sint uobis omnia communia, et distribuatur unicuique uestrum a praeposito uestro uictus et tegumentum, non aequaliter omnibus, quia non aequaliter ualetis omnes, sed potius unicuique sicut cuique opus fuerit. Sic enim legitis in Actibus Apostolorum, quia "erant illis omnia communia et distribuebatur unicuique sicut cuique opus erat". Qui aliquid habebant in saeculo, quando ingressi sunt monasterium, libenter illud uelint esse commune. Sed rursus etiam illi qui aliquid esse uidebantur in saeculo non habeant fastidio fratres suos qui ad illam sanctam (voir note 25) societatem ex paupertate uenerunt. Magis autem studeant, non de parentum diuitum dignitate, sed de pauperum fratrum societate, gloriari. Nec extollantur, si communi uitae de suis facultatibus aliquid contulerunt, nel de suis diuitiis magis superbiant, quia eas monasterio partiuntur, quam si eis in saeculo fruerentur. ...dum tamen unicuique, quod cuique opus est, non negetur".

173. Praeceptum, lignes 13 22: "Qui autem non habebant, non ea quaerant in monasterio quae nec foris habere potuerunt. Sed tamen eorum infirmitati quod opus est tribuatur, etiam si paupertas eorum, quando foris erant, nec ipsa necessaria poterant inuerire. Tantum non ideo se putent esse felices, quia inuenerunt uictum et tegumentum, quale foris inuenire non poterant. Nec erigant ceruicem, quia sociantur eis ad quos foris accedere non audebant, sed sursum cor habeant et terrena uana non quaerant, ne incipiant esse monasteria diuitibus utilia, non pauperibus, si diuites illic humitiantur et pauperes illic inflantur".

174. Praeceptum, lignes 34-35: "Omnes ergo unanimiter et concorditer uiuite, et honorate in uobis inuicem deum cuius templa facti estis". 
sement compte de l'état de santé de tous. Chacun devait être traité selon ses besoins réels ${ }^{175}$. Leur égalité ne devait pas dégénérer en égalitarisme. Mais il reste que le principe fondamental étail l'égalité de tous les "temples de Dieu" et que leur régime alimentaire et vestimentaire était normalement celui des pauvres, sans être celui des miséreux.

Saint Augustin a donc voulu réaliser à l'intérieur du monastère égalité et une fraternité qui n'existaient pas au dehors. A la lumière de nos conceptions actuelles cette action sociale était peutêtre bien limitée. Il reste que saint Augustin a dressé un modèle qui mérite considération et que maintenant encore les monastères pourraient être le terrain d'expériences sociales "d'utilité publique". Dans l'esprit de saint Augustin l'essentiel serait que les monastères fonctionnent réellement comme le col de la tunique de JésusChrist, même si cela ne devait pas attirer l'attention.

IV. Hippone, Fussale, l'Afrique;

Dans la quatrième et dernière partie de son exposé $\mathbf{M}$. Leroux a parlé de "Jean Chrysostome et l'institution monastique". Il y fait vois comment Chrysostome s'est efforcé d'utiliser les moines et les religieuses au service de l'Eglise.

Je retiens de son exposé les passages qui me donnent l'occasion de mettre en évidence des traits comparables dans la vie d'Augustin.

Jean Chrysostome a appelé de nombreux moines au service de l'Eglise de Constantinople, à tous les degrés de la hiérarchie ecclésiastique. Ainsi on trouve dans son entourage épiscopal un prêtre qui est certainement moine, Etienne, les moines diacres Germain

175. Praeceptum, lignes 53-57; 68-77: "Qui infirmi sunt ex pristina consuetudine, si aliter tractantur in nictu, non debet aliis molestum esse nec iniustum uideri, quos facit alia consuetudo fortiores. Nec illos feliciores putent, quia sumunt quod non sumunt ipsi, sed sibi potius gratulentur, quia uolent quod non ualent illi. Sane, quemadmodum aegrotantes necesse habent minus accipere ne grauentur ita et post aegritudinem sic tractandi sunt, ut citius recreentur, etiam si de humillima paupertate uenerunt, tamquam hoc illis contulerit recentior aegritudo, quod diuitibus anterior consuetudo. Sed cum uires pristinas reparauerit, redeant ad feliciorem consuetudinem suam, quae famulos dei tanto amplius decet, quanto minus indigent. Nec ibi eos teneat uoluptas iam uegetos, quo necessitas leuarat infirmos. Illi se aestiment ditiores, qui in sustinenda parcitate fuerint fortiores; melius est enim minus egere, quam plus habere". 
et Cassien, un ascète qui était lecteur et chantre, Eutrope, sans parler de l'archidiacre Sérapion. Comme on le voit, les conseillers habituels de l'évêque de Constantinople étaient des moines, dont certains ont été ordonnés par lui ${ }^{176}$.

Et Augustin? Quand il a pris effectivement la succession de Valerius ${ }^{177}$, après avoir été son "coadjuteur", il est allé habiter la maison épiscopale. Il a voulu que cette maison soit désormais un monastère, le monasterium clericorum d'Hippone. Lui-même et les clercs de son entourage immédiat y vivaient selon le modèle tracé dans le quatrième livre des Actes des Apôtres, versets 31-35: unité des âmes et des coeurs, pauvreté volontaire absolue, prière en commun, annonce de la parole de Dieu, témoignage rendu à la résurrection du Seigneur. Nous le savons par le Sermon 356, prononcé au début de 426. Ce Sermon nous fait connaître les noms de plusieurs moines-clercs qui vivaient avec leur évêque en ce moment: le sous-diacre Patricius, neveu d'Augustin (et homonyme de son propre père), puis les diacres Valens, Faustinus, Severus, Eraclius, plus tard désigné comme successeur d'Augustin ${ }^{178}$, et un certain diacre Hipponensis (adjectif? nom propre?), enfin les prêtres Leporius et Barnabas, sans oublier le prêtre Ianuarius qui venait de mourir en laissant un testament, ce qui était l'occasion de l'enquête dont le Sermon 356 est le compte rendu. Augustin nous dit luimême que cette liste de clercs n'était pas exhaustive-179.

176. Article cité dans la note $3,141-142$.

177. La date de la mort de Valerius est inconnue, mais le 28 août $397 \mathrm{Au}$ gustin a mis, comme Augustinus episcopus, sa signature sous les Actes d'un Concile réuni à Carthage (voir l'édition des Concilia Africae A. 345-A. 525, par C. MUNIER, Corpus Christianorum 149, 49), tandis qu'aucune mention n'y est faite de Valerius. Cela fait penser qu'à ce moment Augustin avait pris effectivement la succession du vieil évêque.

178. Voir les Acta Ecclesiastica du 26 septembre 426, insérés dans la Correspondance de saint Augustin comme Ep. 213: PL 33, 966-968; CSEL 57, 372-379). $\mathrm{Au}$ début de cette "Lettre" se trouve signalée la présence des prêtres Saturninus, Leporius, Barnabas, Fortunatianus, Rusticus, Lazarus et Eraclius. Les deux dernieres étaient encore diacres au début de cette année, d'après le Sermon 356 ne dit rien de Saturninus, Fortunatianus et Rusticus. Ce dernier pourrait être celui qui s'occupait des religieuses dont Felicitas était la praeposita (voir Ep. 210; le début de l'Ep. 211 -il s'agit de la fameuse Obiurgatio- parle du praepositus d'un monastère de religieuses et de pourrait être le même Rusticus). Quant à Saturninus et Fortunatianus, les Acta Ecclesiastica n'affirment nullement qu'ils appartenaient au clergé d'Hippone, bien que cela ne soit pas à exclure. Ces Acta signalent aussi la présence de deux évêques étrangers lors de la désignation d'Eraclius comme successeur d'Augustin, Religianus et Martinianus. Il se pourrait donc que les prêtres Saturninus et Fortunatianus fussent également de passage.

179. Serm. 356, 8 et 15: PL 39, 1577 et 1580 ; éd. C. LAMBOT, 137, et 142. 
Par des textes plus anciens, des Lettres pour être précis, nous connaissons encore d'autres noms: ceux du prêtre Bonifacius et de son "frère" Spes qui désirait être ordonné prêtre également (Lettres. LXXVII et LXXVIII) ${ }^{180}$, celui du diacre Lucillus (Lettre LXXXIV) ${ }^{181}$ et celui du lecteur Antoninus, devenu malheureusement évêque de Fussale (Lettre CCIX) ${ }^{182}$.

Ce monasterium clericorum était la seconde fondation monastique masculine d'Augustin à Hippone. Au lendemain de son ordination sacerdotale il avait déjà fondé un monastère dans le jardin de l'église où il vivait, seul prêtre, avec des moine laïques ${ }^{183}$. Or nous savons par Possidius, le biographe d'Augustin, que ce premier monastère d'Hippone a fourni à l'Afrique une dizaine de pasteurs choisis parmi les meilleurs et les plus savants de ces moines ${ }^{184}$. Possidius lui-même était sans doute du nombre ${ }^{185}$. Ces évêques ont continué ailleurs le genre de vie qu'ils avaient pratiqué autour de saint Augustin à Hippone ${ }^{186}$.

J.-M. Leroux rappelle aussi que Jean Chrysostome a organisé à Constantinople un inmense hospice-hôpital et assuré son fonctionnement par l'intermédiaire d' "ascètes" médecin, infirmier et cuisinier. Il était symptomatique, nous dit M. Leroux, que Jean Chrysostome a confié la direction de cet établissement à deux prêtres qui étaient vraisemblablement moines eux-mêmes. Cela déterminait clairement la responsabilité de l'institution ecclésiale par rapport à cet hôpital ${ }^{187}$.

Possidius nous fait comprendre, quant à Augustin, que celui-ci n'avait pas l'âme d'un bâtisseur. Mais tout en freinant des zèles qui étaient trop impétueux à son gré, il n'empêchait pas ceux qui étaient différents de lui de réaliser des construction ${ }^{188}$. Le Sermon

180. PL 33, 266-272; CSEL 34.2, 329-245.

181. PL 33, 294-295; CSEL 34.2, 392-393.

182. PL 33, 953-957; CSEL 57, 347-373.

183. POSSIDIUS, Vita s. Aug. 5: PL 32, 57; éd. M. PELLEGRINO, 52; Serm. 355, 2: PL 39, 1569-1570; éd. G. LAMBOT, 125-126.

184. POSSIDIUS, Vita s. Aug. 11, 1-3: PL 32, 42; éd. M. PELLEGRINO, 72-74; voir A. Mandouze, Saint Augustin. L'aventure, 229, note 2.

185. A la fin de sa biographie, qui se termine par la morte et les obsèques d'Augustin (430), Possidius affirme qu'il l'avait fréquenté ferme annis quadraginta: PL 32,65 ; éd. $M$. PELLEGRINO, 196. Or la fondation du premier monastère d'Hippone date de 391. D'autre part, Possidius dit (voir la note précédente) qu'il avait connu personnellement la dizaine de moines d'Hippone qui étaient devenus évêques ou clercs. Quant aux autres noms, voir la note précédente.

186. POSSIDIUS, Vita s. Aug., 11, 4: PL 32, 42; éd. M. PELLEGRINO, 74.

187. Article cité dans la note $3,141$.

188. POSSIDIUS, Vita s. Aug., 24, 13: PL 32, 54; éd. M. PELLEGRINO, 130. 
356, dont nous avons déjà parlé, en signale plusieurs, constructions d'églises et de monastères, mais aussi, ce qui nous intéresse plus particulièrement ici, la construction d'un xenodochium ${ }^{189}$. d'un hopis ce-hôpital précisément ${ }^{190}$. C'est le prêtre Leporius qui l'a bâti, mais saint Augustin affirme que cette fois-ci l'initiative en était venue de lui-même: Ego illi iniunxi, ego iussi ${ }^{191}$. Malheureusement nous ne sommes pas informés à propos des dimensions du xenodochium d'Hippone, ni sur son fonctionnement. Mais le seul fait de son existence constitue un parallèle intéressant par rapport à ce que nous est dit à propos de la ville épiscopale de Jean Chrysostome.

M. Leroux nous dit aussi que Jean Chrysostome a envoyé des missionnaires chez les Goths nomades en Scythie et que cette mission était exclusivement composée de moines. Il en est allé de même en Phénicie. Ce sont même de véritables monastères qui prennent en charge des missions. Dans le même contexte l'auteur parle encore de la Perse, des Isauriens et des païens d'Arabissos. Jean Chrysostome s'inquiète partout de l'extension du royaume de Dieu, et pour propager la bonne nouvelle il compte en premier lieu sur les moines ${ }^{194}$.

Saint Augustin, lui, fait davantage l'impression d'un évêque exclusivement diocésain et régional. La géographie eclésiastique y était peut-être pour quelque chose. Mais à l'intérieur de son territoire des attitudes "missionnaires" ne lui étaient nullement étrangères. Il s'est très consciencieusement inquiété des "allophones" de son diocèse, de ces gens qui comprenaient mal la langue latine de leur évêque et de la grande majorité de son clergé.

Dans sa Lettre LXXXIV à l'évêque Novatus de Sitifis (actuellement Setif) Augustin fait savoir au destinataire qu'il refuse de lui envoyer le diacre Lucillus, qui était pourtant le propre frère de Novatus. Augustin avait besoin de lui dans le diocèse d'Hippone où beaucoup de gens ne comprenaient pas le latin, contrairement à ce qui se passait à Sitifis. Il est évident que Lucillus savait une autre

189. Serm. 356, 10 : PL 39, 1578; éd. C. LAMBOT, 138-139.

190. A propos des xenodochia voir G. UHLHORN, Die christliche Liebestätigkeit in der alten Kirche 2 , I (Stuttgart, 1882), 316-331; W. SCHONFELD, "Die Xenodochien in Italien und Frankreich im frühen Mittelalter": Zeitschrift der Savigny-Stiftung für Rechtsgeschichte (C: Kanonistische Abteilung) 43 (1922) 154. G. UHLHORN (p. 322) souligne le caractère global du terme: "...Fremde wurden hier beherbergt Bettler fanden ein Unterkommen, Kranke wurden verpflegt".

191. Serm. 356, 10: PL 39, 1578; éd. C. LAMBOT, 138.

192. Article cité dans la note $3,143$. 
langue que le latin, la Punica lingua sans doute, et que pour cette raison sa présence à Hippone était hautement souhaitable ${ }^{193}$.

Le même souci s'est manifesté dans le malheureux cas de l'évêque Antoninus de Fussala. Nous en connaissons les détails par la Lettre CCIX de saint Augustin, adressée au pape Célestin (422-432). Fussala appartenait au diocèse d'Hippone, mais jusqu'à une date récente toute la population avait été donatiste. Elle était devenue catholique et faisait désormais plus directement partie du troupeau d'Augustin. Malheureusement Fussala se trouvait à quarante milles d'Hippone, si bien qu'il était difficile pour l'évêque de s'occuper effectivement de cette partie de son diocèse. C'est pourquoi il avait décidé d'installer un évêque à Fussala. Les gens de cet endroit parlant la Punica lingua, le choix d'Augustin était tombé sur prêtre du monasterium clericorum qui était un homme compétent et qui parlait cette langue. Augustin avait demandé au primat de Numidie de venir à Fussale afin de procéder à la consécration épiscopale de ce prêtre, dont nous ignorons le nom. Le primat s'était déplacé. Augustin était venu également. Mais l'heure de la consécration arrivée, le prêtre refusa absolument de prendre en charge l'Eglise de Fussala. Augustin aurait mieux fait de remettre à plus tard la solution de ce difficile problème, mais il ne voulait pas décevoir son primat. Il était accompagné d'un autre membre du monasterium clericorum, un jeune lecteur portant le nom d'Antoninus. Dans les circonstances données, il est probable qu'Antoninus parlait la $P u$ nica lingua également. Il était peut-être l'interprète d'Augustin à cette occasion. Devant le refus du prêtre, Augustin a présenté Antoninus à la population de Fussala et, se fiant au jugement d'Augustin, ces malheureux l'ont accepté comme leur évêque. Antoninus a été consacré immédiatement ${ }^{194}$. Mais par la suite il semble avoir bu copieusement du lait de ses brebis et s'être bien habillé de leur laine, pour employer les termes d'Ezéchiel que nous avons rencontrés plus haut en lisant le Sermon 46 de pastoribus. Tant il est vrai qu'Antoninus s'est fait la réputation d'un véritable tyran malhonnête ${ }^{195}$.

Saint Augustin explique tout cela au pape Célestin et se sen-

193. Ep. 84, 2: PL 33, 294-295; CSEL 34.2, 393.

194. Ep. 209, 2: PL 33, 953-954; CSEL 57, 34-349. A propos de la Punica lin$g u a$ voir A. MANDOUZE, oeuvre citée dans la note 184, 73-74, note 2.

195. Ep. 209, 4: PL 33, CSEL 57, 349. 
tant responsable de la pénible tournure que cette histoire avait prise, il lui dit qu'il pense à présenter sa démission comme évêque d'Hippone, si la situation n'allait par s'améliorer ${ }^{196}$.

Mais ce qui nous intéresse surtout en ce moment c'est que saint Augustin a voulu mettre à la tête de l'Eglise de Fussala un prêtre du monasterium clericorum d'Hippone qui parlait la langue de ses futurs diocésains. Les Lettres LXXXIV et CCIX nous démontrent qu'Augustin, ce moine devenu prêtre et évêque, entendait utiliser des moines à des tâches qui étaient missionnaires à leur façon.

LUC VerheiJen, OSA.

Paris

196. Ep. 209, 10: PL 33, 956; CSEL 57, 352-353. 